\title{
Charles Fourier
}

\author{
(1772-1837)
}

Philosophe français.

\section{Du libre arbitre}

(Première édition, 1841)

\author{
Édition des Saints Calus \\ Bordeaux, Paris, Barcelone \\ 2003
}

\author{
Un document produit en version numérique \\ par Jean-Marc Simonet, bénévole. \\ Courriels: jean-marc_simonet@uqac.ca
}

Dans le cadre de la collection: "Les classiques des sciences sociales"

Site web: http://www.uqac.ca/Classiques_des_sciences_sociales/

Une collection développée en collaboration avec la Bibliothèque Paul-Émile-Boulet de l'Université du Québec à Chicoutimi Site web: http://bibliotheque.uqac.ca/ 
Cette édition électronique a été réalisée par Jean-Marc Simonet, bénévole, professeur des universités à la retraite, Paris.

Courriel : jean-marc_simonet@uqac.ca

À partir du livre :

\begin{tabular}{|c|c|}
\hline CHARLES FOURIER & $\begin{array}{c}\text { CHARLES FOURIER } \\
(1772-1837)\end{array}$ \\
\hline 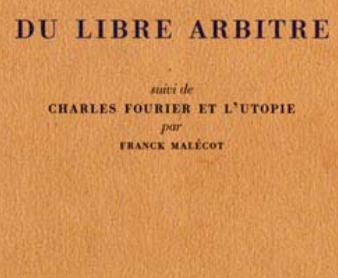 & $\begin{array}{c}\text { DU LIBRE ARBITRE } \\
\text { (première édition,1841) } \\
\text { édition établie par } \\
\text { PASCAL FIÉVÉ }\end{array}$ \\
\hline 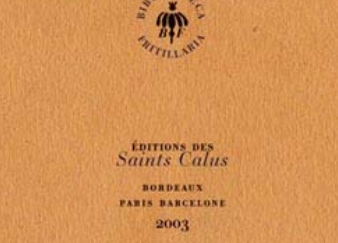 & $\begin{array}{c}\text { Édition des Saints Calus, Bordeaux, } \\
\text { Paris, Barcelone, 2003, } 142 \text { pages }\end{array}$ \\
\hline
\end{tabular}

Polices de caractères utilisées :

Pour le texte: Times New Roman, 14 points.

Pour les citations : Times New Roman 12 points.

Pour les notes de bas de page : Times New Roman, 12 points.

Édition électronique réalisée avec le traitement de textes Microsoft Word 2004 pour Macintosh.

Mise en page sur papier format : LETTRE (US letter), 8.5' x 11'”)

Édition numérique réalisée le 30 mai 2009 à Chicoutimi, Ville de Saguenay, province de Québec, Canada. 
TABLE DES MATIÈRES

$\underline{\text { Avertissement aux lecteurs (1841, extraits) }}$

\title{
DU LIBRE ARBITRE
}

\author{
Antienne \\ Division du libre arbitre en actif et en passif \\ Des cas de perclusion du libre arbitre \\ Du libre arbitre de Dieu et de l'homme \\ $\underline{\text { Du libre arbitre de l'homme, en simple et en composé ; en positif et en }}$ \\ négatif \\ Du libre arbitre en carrière rétrograde \\ Du libre arbitre en carrière extrograde \\ Classement des libertés vraies et illusoires \\ Compétence théologique \\ Compétence philosophique \\ $\underline{\text { Compétence des deux sciences }}$
}




\section{AVERTISSEMENT AUX LECTEURS DU LIBRE ARBITRE (1841, extrait.)}

Le Traité du Libre Arbitre ne figure pas dans la première édition du Traité de l'Unité universelle: c'est le premier des manuscrits de Fourier livré à l'impression depuis la mort de l'auteur.

Les cahiers laissés par Fourier ne sont en général que des travaux de premier jet qu'il condensait et ordonnait lorsqu'il devait les publier. Un assez grand nombre de ces manuscrits sont d'une date antérieure à celle où a paru la première édition du Traité de l'Unité universelle (1822).

Le Traité du Libre Arbitre est de ce nombre. 


\section{ANTIENNE}

DE TOUTES les bévues de notre siècle, il n'en est pas de plus funeste que l'esprit de liberté, bon et très louable abstractivement mais si mal dirigé, en application, qu'il a rallié aux bannières du despotisme ceux même qui avaient penché pour la liberté. Une fâcheuse épreuve a démontré qu'il n'y a qu'illusion et péjorantisme dans ces belles théories.

Pourquoi donc les nations CIVILISÉES ne sont-elles pas aptes à jouir d'un bien qui est l'objet des désirs collectifs et individuels ? Question bien digne de notre attention ! C'est la première qui doit nous occuper dans une analyse de la CIVILISATION. Il faut d'abord démontrer dans le mécanisme CIVILISÉ aberration spéculative, ignorance des conditions de liberté collective et individuelle. Ce sera l'objet de la $1^{\text {re }}$ section, d'où nous passerons à l'analyse des erreurs pratiques et des ressorts dont le jeu mal dirigé condamne la société CIVILISÉE au rôle de servitude permanente, quelque forme qu'elle puisse donner à ses codes et institutions, en pays populeux, l'exception ne portant que sur pays neufs.

L’asservissement du CIVILISÉ, même dans les républiques où il est souvent bien plus esclave que sous un roi, témoins les oligarchies de Venise, Berne et Fribourg, cet asservissement, dis-je, est si bien constaté que toute preuve à cet égard serait superflue; mais il reste à l'orgueil quelque retranchement où il fait résistance, et à défaut de libertés politiques et matérielles, il se targue de quelques libertés spirituelles et notamment du libre arbitre, que chacun s'accorde à admettre, pour obvier aux croyances de prédestination et de fatalisme qui, faisant de l'homme un automate, élèvent le crime au niveau de la vertu. Je ne prétends pas traiter ces abstruses questions, mais seulement la partie qui est relative à l'attraction. 
Lorsque le roi Louis XVI, bloqué aux Tuileries par les conventionnels, était obligé de signer tous les décrets qu'on lui proposait, une gravure le représenta enfermé dans une prison et passant sa main à travers les barreaux pour écrire : « Je suis libre. »

Telle est l'indépendance dont nous jouissons en CIVILISATION sur l'exercice de nos passions : nous sommes libres de souffrir, mais non pas libres de nous plaindre. L'animal a non seulement le droit de jouir sans qu'on lui intente un procès de larcin ou d'adultère, mais il a le droit de se plaindre si on l'empêche de jouir. Un chien renfermé a le droit de hurler dans sa cage ; un conscrit n'a pas le même privilège, et il faut qu'arraché par les sbires à sa famille et à ses habitudes, il s'écrie comme Louis XVI : « Je suis libre ! Je me pâme d'amour pour la personne sacrée de Bonaparte ! Je jouis de mon libre arbitre ! », etc.

Tels sont les arrêts de la philosophie et de la théologie ; on ne les amènera pas à confesser que le CIVILISÉ soit un vil esclave, bafoué dans ses vertus malheureuses, exalté dans ses crimes heureux : c'est, disent-elles, un être qui a le libre arbitre d'opter entre le bien et le mal. Entre-temps, on prend de bonnes mesures pour qu'il n’hésite pas sur l'option.

S’il est une question à laquelle on doive appliquer le précepte de Bacon, refaire l'entendement humain et oublier tout ce que l'on a appris, c'est bien celle du libre arbitre. Il faut toute l'effronterie de nos sophistes pour prétendre que l'homme est libre d'opter entre le bien et le mal quand on lui persuade que, s'il opte pour ce qu'on appelle mal, il sera torturé en ce monde par les bourreaux ou les sicaires de la philosophie ; en l'autre monde par les démons ou sicaires de la théologie. L'animal même, quoique dépourvu de raison, n’oserait pas en pareille chance opter pour le prétendu mal.

Placez un chien affamé près d'un pâté, son premier mouvement sera de commettre le mal, voler et manger l'objet convoité ; mais faiteslui voir le fouet suspendu sur sa tête, le pauvre animal s'éloignera et semblera vous dire : "Si j'étais libre, je mangerais le pâté, mais tu me rouerais de coups ; j'aime mieux souffrir la faim. »

Tel est le libre arbitre dont jouit l'homme CIVILISÉ et BARBARE: il est libre d'opter pour le plus ou le moins de privations et de supplices, et non pour le bien-être dont il voit les éléments autour de lui. S’il ré- 
pugne à être pendu, il peut opter pour le petit inconvénient de se laisser mourir de faim, selon les principes de la morale du commerce et de la Charte qui condamnent le pauvre au gibet quand il ose demander du travail, du pain et un minimum social.

La législation répondra qu'on ne voit guère de gens mourir de faim. N'en vît-on qu'un par siècle, comme ceux de Seignelay et Bruxelles, ce serait assez pour condamner la législation qui n'assure point de minimum au pauvre et prétend qu'il jouit du libre arbitre. D'ailleurs, souffrir la faim, les privations, n'est-ce pas être victime comme celui qui meurt de faim : il n'y a de différence que celle d'un long supplice à un trépas subit.

Les deux sciences philosophie et théologie, qui assurent au pauvre tant de bonheur, s'affublent des masques de balance, contrepoids, équilibre, garantie, perfectibilité. On peut comparer ces verbiages à celui des jacobins de 1793, qui, à chaque mot, faisaient retentir les principes, les faits, la justice et le bien de la patrie, etc. Admirable chose en CIVILISATION que l'abus des mots! Lorsque Condillac nous dit: "Les mots sont les véritables signes de nos idées », n’aurait-il pas mieux fait de dire : "Les mots sont les véritables masques de nos idées »?

Venons au sujet. Il s'agit d'établir que si l'homme ne jouit pas du libre arbitre, Dieu n'en jouit pas non plus sur notre globe. En effet l'attraction vient de Dieu, et si elle est étouffée par $1 / 8^{\mathrm{e}}$ de privilégiés comprimant les $7 / 8^{\mathrm{e}}$, les salariés, esclaves et autres classes, l'impulsion de Dieu est réellement et complètement entravée, puisque les $7 / 8^{\mathrm{e}}$, en calcul de mouvement, signifient l'ensemble, et que l'exception de $1 / 8^{\mathrm{e}}$ confirme la règle. Ce n'est donc pas l'homme seul, mais Dieu et l'homme qui sont privés du libre arbitre sur tout globe où l'attraction est entravée. Cette privation est COMPOSÉE et non pas SIPMLE, puisqu'elle s'étend aux deux agents primordiaux du mouvement social, à Dieu et à l'homme.

Nous avons donc sur le libre arbitre un double problème à résoudre. Il faut garantir les libertés de Dieu et celles de l'homme, assurer le concours des deux libertés, leur action unitaire, par l'essor de l'attraction.Tel est le vrai sens de la question dont nos philosophes et nos théologiens n’ont envisagé que la moitié : car ils n’ont songé 
qu'au libre arbitre de l'homme, sans acception de celui de Dieu qui est opprimé sur un globe si l'attraction n'y jouit pas du plein exercice.

Opprimer Dieu! Qu'on ne s'étonne pas de cette expression. Les théologiens prétendent bien que l'homme peut tenter Dieu, c'est-àdire lui faire commettre le mal : car la tentation suppose la chance de faire succomber l'individu tenté. Mon assertion n'est point incongrue comme celle des théologiens; je prétends seulement que l'homme peut entraver Dieu dans ses mesures bienfaisantes, en paralyser l'effet et tomber dans le malheur en voulant se diriger sans l'intervention de Dieu. Tel est sur notre globe le résultat du défaut de libre arbitre. Il n'y existe en aucun sens : deux circonstances concourent à nous en priver. Ce sont l'ignorance des lois de la nature, et la perversité des sciences qui s'en disent interprètes. Elles attribuent le défaut de libre arbitre au despotisme des gouvernements. Rien n'est plus erroné, et la preuve en est que les philosophes sont encore plus despotes que les princes quand on leur confie l'administration. Il est donc très faux que la philosophie ait l'intention sincère de rendre aux nations des libertés.

Du moment où l'homme recouvrerait l'usage du libre arbitre ou seulement du gros bon sens, il ne manquerait pas de reconnaître qu'il est dupe des deux sciences qu'il a choisies pour guides, et s'il ne s'en est pas encore aperçu, il faut que des incidents quelconques entravent chez lui le plein exercice du jugement ; c'est par l'examen de ces entraves que nous allons commencer. 


\section{DIVISION DU LIBRE ARBITRE EN ACTIF ET PASSIF}

Nous AVONS à démontrer que le libre arbitre, dans l'état CIVILISÉ, est illusoire, passif et subordonné aux impulsions de l'intrigue ou du préjugé, enfin dangereux pour les masses comme pour les individus, parce qu'il n'est communément qu'une suggestion plus ou moins trompeuse, sauf des exceptions si rares qu'elles confirment la règle et réduisent le prétendu libre arbitre des CIVILISÉs en privation réelle.

Pour expliquer cette privation, il faut se rattacher à l'influence des deux causes assignées en Antienne : ignorance des lois de la nature et perversité des sciences qui s’établissent nos guides. Aucune des deux causes ne pourrait, isolément, opérer le mal dont il s'agit; mais les deux causes réunies y réussissent pleinement.

Leur action combinée prive le libre arbitre de facultés actives ou directes et le réduisent an rôle passif ou subordonné. Cet exercice illusoire est un état de nullité, ainsi qu'on va en juger par la définition de l'actif et du passif.

Pour opter activement sur le bien ou le mal, il faut être capable de juger, et celui qui manque soit de judiciaire, soit de lumières spéciales, n’a plus qu'un arbitrage subordonné aux impulsions étrangères, comme le serait un enfant de dix ans auquel on donnerait sa fortune à gérer. Il serait, à l'instant, cerné d'intrigants, dupé, ruiné en cédant à leurs impulsions. Son intention ne serait pas de se faire spolier : il le serait pourtant, parce qu'un libre arbitre qui n'est que passif et non étayé de lumières et de judiciaire équivaut à une servitude réelle ; et l'arbitrant, malgré sa faculté d'option, n'est que l'instrument mis en jeu par les opinions d'autrui. Il n'a plus de liberté directe : on en voit la preuve dans les rois faibles que l'histoire nomme fainéants. Ils font dans quelques occasions le bien sans le savoir : leur liberté en administration n'étant point un arbitrage du bien ou du mal, mais seule- 
ment une fantaisie, réglée sur les suggestions accidentelles. Or, par le mot libre arbitrage, ou option pour le bien ou le mal, on n'entend pas licence, fantaisie aveugle, mais jugement éclairé, motivé aussi fixement que celui du géomètre.

Toute autre liberté d'arbitre n'est qu'un exercice de la déraison et de l'arbitraire opposé à l'arbitrage. Or, nous avons à spéculer sur l'extirpation de l'arbitraire et l'instauration de l'arbitrage ou détermination libre, fondée en théorie sur l'évidence de justice, et en pratique sur l'utilité d'application. Telle est la faculté dont l'homme CIVILISÉ est privé par double cause, par l'ignorance des règles de justice ou lois de la nature, et par l'influence des deux sciences trompeuses, la philosophie et la théologie, qui mettent leurs insinuations arbitraires à la place de la justice et de la nature.

Je crains de fatiguer les lecteurs par ces principes abstraits, et je vais recourir à une comparaison familière, d'où je passerai à l'application.

Représentons la société BARBARE par un aveugle égaré pendant la nuit. Il a contre lui double cécité : le défaut d’yeux et les ténèbres qui l'empêchent de réclamer assistance. Je fais allusion par cette double cécité à l'ignorance des lois de la nature et au despotisme, qui, chez les barbares, s’oppose à tout progrès des lumières.

A la pointe du jour (c'est l'origine de la CIVILISATION), l'aveugle, après de vains tâtonnements, se trouve engagé dans une prairie marécageuse; ses cris excitent l'attention de deux hommes placés aux deux rives opposées du marécage. Tous deux s'offrent à le diriger par leurs avis, mais sans s'exposer avec lui dans les marais. Tels sont nos philosophes et nos théologiens, qui veulent bien diriger les peuples dans le dédale de la pauvreté, mais non pas s’y engager eux-mêmes.

Le premier guide, la théologie, crie à l'aveugle : " Tirez à droite et venez vers moi, c'est le bon chemin. » L'aveugle obéit, mais il se trouve plus engagé dans la fange : il n'en avait qu'à mi-jambe, il enfonce jusqu'aux genoux et recule précipitamment.

« On vous trompe, lui crie l'autre guide, la philosophie, tirez à gauche, venez de mon côté, c'est le bon chemin. "L'aveugle s’y engage et retombe jusqu'aux genoux dans la fange ; il rétrograde une 
seconde fois, puis après de nouveaux essais, selon les avis de l'un et de l'autre, s'embourbant de plus en plus et ne sachant plus à qui des deux se fier, il reste immobile au plus épais du marécage. Tel est l'effet des lumières acquises depuis le POINT DU JOUR SOCIAL ou origine de la CIVILISATION. Ces lumières en sens philosophique ou théologique sont un cercle vicieux si bien constaté, les intrigues jésuitiques et clubiques, les empiétements du sacerdoce et du philosophisme ont tellement fatigué les souverains et répandu tant de défiance, que chacun flotte entre les deux flambeaux sans confiance pour aucun. Revenons à l'aveugle embourbé par ses guides.

Un troisième s'avance et lui crie : "Puisque ces deux hommes vous ont égaré, et qu'en vous portant vers l'un ou vers l'autre vous tombez également dans la fange, n'écoutez aucun des deux, revenez sur vos pas dont je vois la trace, et quand vous aurez regagné la terre ferme, je vous conduirai à mon logis où je vous ferai l'opération de la cataracte ; après quoi vous aurez pour vous diriger les deux moyens qui vous manquent, le positif ou jouissance de la lumière, et le négatif ou absence des guides maladroits qui vous égarent. »

Tel est en substance l'avis donné à l'esprit humain dans le Traité de l'Harmonie. On lui prouve que ses deux guides le jettent de Charybde en Scylla, qu'en suivant leurs ineptes conseils il ne jouit que d'un libre arbitre illusoire car son intention est d'atteindre au bonheur, et les deux sciences ne le conduisent qu'à l'indigence. Il doit débuter par revenir sur ses pas, se dégager du bourbier de 600000 volumes où sa raison est enfoncée. Après qu'il aura, selon l'avis de Bacon, oublié de ce fatras tout ce qu'il en a appris, refait son entendement en élaguant les insinuations des deux sciences, leurs prestiges sur une destinée malheureuse et limitée aux échelons CIVILISÉ, BARBARE et SAUVAGE, on pourra lui lever la cataracte, l'initier à la théorie des autres voies sociales, à l'étude de la nature ou attraction, foulée aux pieds par les deux sciences. L'homme, après ce traitement, aura surmonté les deux obstacles qui le réduisent au libre arbitre passif, qui sont l'ignorance des lois de la nature et la perversité des guides qui s'emparent de lui dans son aveuglement. Jusque-là sa raison n’est qu'un dédale et son arbitrage qu'une illusion.

Passons au rôle actif ou exercice direct du libre arbitre, qui suppose l'indépendance des préjugés et les connaissances exactes sur la 
DESTINÉE. Quelle règle devra suivre l'homme pourvu de ces nouvelles lumières ? La règle de s'astreindre aux lois de la nature en développant les cinq passions SENSITIVES et les quatre AFFECTIVES, selon l'ordre indiqué par les trois DISTRIBUTIVES. Il ne peut pas exister de bonheur collectif et individuel hors de cette méthode, aucun être ne pouvant être heureux positivement sans l'essor de sa nature ou développement de ses attractions. Nous ne pouvons donc nous flatter de connaître les lois de la nature passionnelle qu'autant que nous aurons découvert un moyen de développer dans cet ordre nos douze passions, et nous ne pouvons arriver à cette découverte qu'en oubliant les dogmes de nos 600000 volumes philosophiques et théologiques, plus ou moins opposés à l'essor des passions.

Parvenus à ce nouveau mécanisme social, notre libre arbitre sera actif par deux raisons, parce qu'il ne sera impulsion d'aucune secte mais de Dieu par l'attraction, de nous-mêmes par la raison convergente ou coïncidente avec les lois de la nature et de ses harmonies matérielles. Ce n'est qu'en marchant dans cette voie que nous pourrons nous flatter d'un exercice positif de la raison, qui tendra alors à raffiner continuellement nos plaisirs. Elle est négative dans l'ordre actuel, où ses impulsions les plus sages ne nous poussent qu'au mal et ne remplissent aucunement leur destination qui est de nous diriger dans les plaisirs, d'en étendre le cercle, d'en accroître l'intensité par l'emploi des trois passions distributives.

Concluons en récapitulant sur cette thèse qu'il n'existe pas aujourd'hui de libre arbitre, et que le rôle passif auquel il est réduit est une liberté illusoire, ainsi qu’il a été précédemment démontré.

Voilà pour la théorie. Il nous reste à l'appliquer à quelques détails de la pratique CIVILISÉE, aux formes vicieuses que prend le libre arbitre dans cette société, où il ne nous pousse qu'à l'encontre de la DESTINÉE, qu’aux mécanismes :

- simple au lieu de composé ;

— négatif au lieu de positif ;

— passif au lieu de l'actif ;

— qu'au duplicisme an lieu de l'unitéisme ;

— qu'au subversif au lieu de l'harmonique. 
Nous allons débuter par une thèse mixte sur l'engorgement ou perclusion du libre arbitre; de là nous passerons à ses développements CIVILISÉS, où nous trouverons en tout sens les vices dont nous venons de l'accuser. 


\section{DES CAS DE PERCLUSION DU LIBRE ARBITRE}

NouS ALLONS sonder la raison sur un point des plus délicats, et lui réitérer une de ces accusations que lui firent Newton et Copernic, celle d'être à rebours du bon sens. Il faut se garder là-dessus des répliques à la française, de la manie de renvoyer l'accusation à son auteur, en disant que c'est lui sans doute qui n'a pas de bon sens. Ces ripostes sont pure duperie, et pour preuve il n'est rien de plus heureux pour le genre humain que les débats où on lui démontre qu'il manque de raison depuis plusieurs mille ans. C'est ce que lui ont prouvé Copernic, Newton, Galilée, Colomb et tant d'autres. N'est-il pas à souhaiter qu'on lui donne sans délai pareil camouflet sur toutes ses erreurs ? Et la vérité tant désirée peut-elle apparaître sans que sa lumière soit un affront pour le préjugé qu'elle dissipe ? C’est donc se déclarer ennemi de la vérité que de s'offenser de ce qu'un homme débute par dire que le genre humain a manqué de sens commun sur telle question. Ce prétendu outrage, s'il choque les petits esprits, doit être un sujet de joie pour les amis des lumières. J'oserai donc leur dire, sans crainte de les indisposer, que l'esprit humain s'est à plaisir perclus l'entendement et fermé la voie des découvertes, quand il a méconnu qu'il doit entrer en participation de puissance avec Dieu, en vertu du titre d'infiniment petit et dernier en chaîne d'HARMONIE.

Pour bien nous convaincre de sa passivité dans l'état actuel, observons que les deux causes qui le réduisent au rôle passif (ignorance des lois de la nature et perversité de nos deux guides) ont souvent assez d'influence pour perclure de jugement l'humanité tout entière sur diverses questions. C'est ce qui arrive de toutes les questions de DESTINÉE et d'ATTRACTION, et même de beaucoup d'autres sur lesquelles le genre humain, dans sa pleine liberté, tombe en crétinisme spécial. 
Par exemple : quand le genre humain tout entier s'insurgeait contre Colomb qui annonçait un nouveau continent, quand la philosophie et la théologie l'accablaient de leurs foudres, n'y avait-il pas sur ce sujet perclusion de jugement chez la masse entière ? Il est clair aussi que tout le genre humain déraisonnait, qu'aucune de ses répliques ne pouvait être valable. Cependant il n'avait pas l'intention de se tromper lui-même, d'être rebelle à tout indice de lumière ; il l'était pourtant et il l'est de même aujourd'hui sur la DESTINÉE heureuse et l'ISSUE DES LIMBES. Il est donc des cas où l'influence des deux sciences, jointe à l'ignorance des lois de la nature, amène le genre humain à une perclusion totale du libre arbitre, puisqu'on entend par arbitrage libre la faculté de porter un jugement sain et dégagé de suggestions mensongères.

Appellera-t-on préjugé cette erreur collective, ce crétinisme universel ? Le mot de préjugé ne suffit point à la qualifier. On entend par préjugé une opinion sans examen. Il s'agit ici d'une rébellion réfléchie et voulue par tout le genre humain, contre des vérités dont l'examen et l'épreuve tendent à la satisfaction collective et individuelle. Quand les deux sciences perfides ont assez d'empire pour le liguer tout entier contre son propre vœu, il ne jouit plus de sa liberté de jugement, mais seulement d'une libre adhésion à l'hébétement intellectuel, et il y a perclusion du libre arbitre qui suppose entremise de raison, et préalablement existence de raison. Distinguons entre l'entrave et la privation : un homme que la peur a glacé et qui croit voir un fantôme, n'a pas la force d'avancer ; un tel homme n'est qu'entravé et non pas paralysé comme celui qui serait perclus des deux jambes. Même distinction doit être faite sur les facultés mentales. Elles ne sont qu'entravées quand un préjugé les arrête; mais elles sont percluses quand l'obstacle naît d'une force presque insurmontable, d'une vraie paralysie, comme le prestige philosophique et théologique contre la découverte des DESTINÉES et l'ISSUE DE CIVILISATION.

Tel est le degré d'abrutissement où nous ont conduits la philosophie et la théologie sur toutes les questions relatives à la DESTINÉE. L'esprit humain, sur ces questions, n'est pas maître de douter, éprouver, vérifier. Le despotisme philosophique l'a façonné à repousser obstinément toute voie de vérification, tout indice de découverte : aussi l'esprit humain est-il novice jusqu'au crétinisme sur toutes les bran- 
ches d'études de la DESTINÉE. Appuyons ceci d'un indice tiré de ces considérations.

Je choisis la destination des infiniment petits et leurs rapports avec les infiniment grands, thèse qui comprend les rapports sociaux de l'homme avec Dieu, tous deux étant les extrêmes de petitesse et de grandeur en chaîne d'HARMONIE.

Le nom de Dieu n'inspire malheureusement parmi nous que terreur chez les opprimés, dédain chez les oppresseurs. Aucune de ces deux classes ne peut s'élever à juger sensément de Dieu, à voir en lui un protecteur, un ami qui veut nous associer à sa puissance, et l'on peut dire que, sur ce sujet, les savants mêmes ne jouissent pas de leur libre arbitre. L'aspect des misères du monde, l'esprit de rébellion secrète qu'elles excitent chez l'homme juste, s'opposent à un rapprochement amical entre la créature et le Créateur. Si l'on n'arrive pas à cet esprit, il est impossible de juger sainement des vues de Dieu sur notre sort.

On a voulu nous inspirer pour lui une tendresse filiale, sous prétexte qu'il est le père de la nature. Fausse application! Car l'autorité paternelle, parmi nous, est nécessairement coercitive et non pas amicale. Dans l'HARMONIE, au contraire, cette autorité n'existe pas et se transforme en adulation, en déférence continuelle du père pour l'enfant : or si nous ne devons pas, comme l'insinuent nos sciences, nous ravaler devant Dieu, nous ne devons non plus nous croire ses supérieurs. Le véritable ton de nos relations avec lui doit être l'amitié. Si j'en juge de la sorte, c'est que, mieux instruit qu'un autre de ses desseins sur nous, je vois que toutes ses dispositions sont celles d'un ami libéral, généreux, qui veut partager franchement avec nous son bonheur et son empire sur le mouvement. Tel est l'esprit dont il convient de se pénétrer pour bien concevoir le CALCUL DES DESTINÉES.

Nos deux guides, philosophie et théologie, nous polissent aux deux excès opposés à cet esprit, et d'abord la théologie nous inspire, dans nos rapports avec Dieu, la stupeur du villageois qui, introduit à la table d'un empereur, ose à peine goûter des mets qui lui sont présentés : tout préoccupé de l'idée de la puissance du monarque, il perd l'usage de ses facultés mentales et devient le plus insipide personnage ; mais s’il écoute l'esprit philosophique, il insultera l'empereur, lui débitera quelque bouffissure démagogique, de la force de celle-ci : " Pour être 
plus qu'un roi tu te crois quelque chose ! » Qu'arrivera-t-il de ces deux excès ? Que ni le souverain ni le villageois n'auront joui de l'agrément que cette rencontre pouvait réciproquement leur procurer si le villageois, évitant ces deux excès, eût conservé dans le dialogue son aplomb et le ton aisé qu'il prend avec un homme égal à lui ou légèrement supérieur.

Nous tombons dans les deux excès que je viens de décrire. Il en résulte des deux côtés une perte réelle et pour Dieu et pour l'homme. Celui-ci, à la vérité, éprouve le plus grand dommage ; mais Dieu perd de son côté par la privation de régie active sur notre globe et par les retards d'une foule d'opérations matérielles : c'est double entrave, dont la cessation dépend de nous, de notre retour au sang-froid, aux calculs d'attraction, sans lesquels nous ne pouvons ni juger la destination du mouvement, ni procéder à remplir la tâche qui nous est assignée : l'association aux travaux de Dieu et l'initiative en divers emplois.

Mais vous parlez de bouleversements gigantesques, diront les critiques; vous voulez anéantir les trois sociétés CIVILISÉE, BARBARE, SAUVAGE, déplacer les astres et les univers! Pourquoi non, si cela est utile et que l'homme soit réellement appelé à cette coopération ? Voilà sur quoi il faut disserter avec calme : si la prétention est outrée, on s'en convaincra par l'examen des moyens et leur facile épreuve. Après quoi l'on sera à temps de rejeter ces belles espérances. Mais tant que la raison humaine n'osera pas s'élever à l'idée d'association avec Dieu dans la gestion de l'univers, selon la loi du contact des extrêmes, elle n'aura pas le libre arbitre rationnel, ou libre exercice de ses facultés intellectuelles. Un respect stupide pour les grandeurs de Dieu rendra l'homme incapable de juger sa propre grandeur, sa propriété de contact d'extrêmes et participation de régie à titre d'infiniment petit et dernier chaînon d'HARMONIE. Nous n'aurons que de fausses idées sur l'immensité de Dieu si nous pensons qu'elle soit simple ; qu'il ne soit immense qu'en direct et non en inverse, qu'il ne puisse pas attacher d'immenses effets au mouvement d'un atome fonctionnant à propos. Nous-mêmes jouissons de cette propriété, et nous savons au moyen d'une étincelle, faire sauter une ville ; doutera-t-on que Dieu ne puisse faire pour le bon ordre ce que nous savons faire pour le désordre ? Et que dans ces vastes plans, où tout se lie du grand au petit, le déplace- 
ment d'un atome ne puisse devenir l'initiative d'une métamorphose universale?

Lorsqu'un immense feu d'artifice est disposé, et la foule rassemblée pour le voir, à quoi tient le commencement du spectacle ? A une étincelle qui enflammera successivement toutes les pièces, en les supposant liées par des veines inflammables.

Tel est le secret de la destinée actuelle de notre univers : tout y est préparé pour un brillant coup de théâtre dont notre globule doit prendre l'initiative par un grain d'arôme en titre pur qu'il versera au soleil ; mais pour le verser pur, il faut se mettre en mesure de le fabriquer, et en fournir à la planète les moyens longtemps retardés.

« Ne suffit-il pas de Dieu pour opérer ? », diront les théologiens, « et pourquoi entremettre un ver de terre qui n'est que cendre et poussière ? " Cette suffisance de Dieu est une hérésie en MOUVEMENT. C'est donner à Dieu une essence incohérente, des attributions simples ; il veut des associés en tous genres de fonctions et ne fait comme nous emploi du simple qu'en relais du composé : nous devons donc, pour juger sainement de ses opérations, spéculer d'abord sur l'action composée ou intervention de l'associé infiniment petit dans les opérations universales qui tiennent à un effet infiniment petit. Peut-on douter que le coopérateur de Dieu dans ces fonctions minimes ne doive être l'homme ou agent minime d'HARMONIE?

Si les esprits, sous prétexte d'immensité, s'ébahissent à l'idée de ces opérations; si, an lien de raisonner avec calme, ils dissertent comme Don Quichotte frappé de visions gigantesques, on pourra leur reprocher de manquer du libre arbitre rationnel qui ne dépend que d'eux-mêmes et qui est l'opposé de la crédulité, ainsi que je l'ai démontré dans l'exposé des sept conditions à exiger d'un inventeur.

S’ils ne savent pas s’élever à cette indépendance des préjugés ; s'ils dédaignent cette boussole des sept garanties de vérité, ils se privent par là même de leur libre arbitre rationnel, pour se mettre sous le joug des prestiges théologiques et philosophiques. Cependant ils ont des intentions confuses de s'initier à l'étude de la nature et des relations de l'homme avec la Divinité ; jamais ils n'en concevront de justes idées tant qu'ils se refuseront à la théorie d'unité et de contact d'extrêmes entre les infiniments petits et les infiniments grands. 
C'est sur cette vérité que repose le gage des hautes destinées de l'homme et du partage que Dieu lui réserve dans la régie de l'univers. Le reproche de petitesse que la théologie adresse à l'homme est précisément le titre qui nous vaut l'immensité de faveur divine. Quant à la philosophie, qui veut faire de nous des colosses, des titans aptes à opérer en mouvement social ou autre sans l'intervention sociétaire de Dieu, sans le concert avec l'attraction, oracle de Dieu, ses opinions sacrilèges décèlent un dépit caché d'avoir manqué les voies de la nature et le plan de ses prétendus mystères.

Aujourd'hui qu'il est découvert et que les contes de voiles d'airain vont prendre place parmi les contes d'enfants, n’hésitons pas un instant à nous isoler des deux sciences qui nous ont dérobé trois mille ans le secret des lois de la nature et du bonheur social. Sur quelque antre point qu'on veuille les scruter, on les trouvera, comme dans la question du libre arbitre, toujours engagées dans les deux excès sans vouloir se rapprocher ni de la raison ni de la Divinité. Avec un peu de réflexion et en consultant cette analogie qu'ils recommandent sans cesse, ils auraient reconnu que c'est notre petitesse même qui nous garantit plus de contact avec la Divinité que n'en ont les créatures d'ordre supérieur, les planètes, les tourbillons, les univers.

Cette théorie de l'infiniment petit n’a jamais été traitée en sens concret on composé, du matériel et du passionnel. Les physiciens et les géomètres en ont parlé, les uns abstractivement, les autres simplement sans l'étendre au delà des emplois matériels; mais en l'appliquant au passionnel et aux destinées générales, elle devient pour l'homme un palladium de grandeur prochaine, un véhicule vraiment digne d'exalter son génie, dissiper ses défiances de bonheur et le remplir des plus hautes espérances. Une planète, créature infiniment supérieure à nous en intelligence, n'a pas comme nous le gage de suprématie fondée sur le titre d'échelon ultérieur et infiniment petit harmonique, réservé par sa ténuité même à partager avec Dieu l'initiative des plus immenses mouvements de l'univers.

Après ces données sur nos attributions méconnues, procédons à juger ces aristarques pétris de préjugés sur la faiblesse de l'homme, et criant à la démence quand on lui pronostique de hautes destinées. J'ai donné à leur secte le nom d'ESTROPIÉ MENTAL, privé du libre arbitre rationnel. Démontrons. 
Un être n'est-il pas perclus de facultés, au moins en partie, quand il ne s'élève pas au développement matériel ou intellectuel assigné à son espèce ? Par exemple, les esclaves russes, qui ne peuvent pas concevoir ce que c'est que la liberté, ou du moins pas concevoir qu'ils puissent en jouir, ne sont-ils pas en partie perclus d'esprit et comparables à celui qui est paralysé d'un membre?

Même perclusion s'étend à la classe savante de notre globe, sur la question des destinées concrètes en infiniment petit, question aussi neuve pour ces savants que le furent dans le temps celles proposées par Colomb, Copernic et autres. Jamais l'athéisme n'aurait pu germer si l'on avait eu des notions saines sur l'infiniment petit harmonique ; mais tant que les lois de la nature et des DESTINÉES ne sont pas connues, le calcul de l'infiniment petit harmonique ne peut pas être l'objet des études, et de là vient que cette question aujourd'hui est aussi vierge que le Traité entier de l'attraction où elle occupe un chapitre.

Les savants de notre siècle étant privés de cet appui, de cette connaissance des DESTINÉES HARMONIQUES, il n'est pas étonnant qu'ils aient donné dans l'abus de la science et adopté toutes les doctrines honteuses pour l'esprit humain : athéisme, matérialisme, déisme et autres aberrations du génie simple. Jusqu'au jour de résipiscence, on est bien fondé à les comparer en intelligence des DESTINÉES l'esclave qui ne peut pas concevoir que la liberté soit faite pour lui.

Ces préventions contre la haute importance de l'infiniment petit ont dû se communiquer des savants au monde CIVILISÉ dont ils sont précepteurs, et sur les questions de ce genre le préjugé ne permet pas même la discussion. Or, comment qualifier cette paralysie factice en étude des DESTINÉES ? On ne peut pas la nommer aliénation mentale, germe de démence, car rien n'empêcherait que le monde social ne s'en guérît comme d'une paralysie matérielle, et ne secouât tous les préjugés qui l'offusquent en matière de DESTINÉEs: il suffirait qu'un écrivain ou un capitaliste marquant donnât l'élan.

L’humanité ne manque donc point des facultés nécessaires pour sa guérison, mais elle est sur ce sujet percluse du libre arbitre par l'opacité des préjugés dont il lui serait fort aisé de secouer le joug, et qui lui ôtent la faculté de porter des jugements réguliers en matière de 
DESTINÉE. Je viens de le démontrer en traitant du destin des infiniment petits. L'examen de toute autre branche des DESTINÉES nous mènerait à la même conclusion, celle de paralysie intellectuelle du genre humain et absence de libre arbitre sur tout ce qui contredit les dogmes réunis de la philosophie et de la théologie, vraies mégères qui feignent de se vouer à la recherche de la vérité pour mieux asservir l'esprit humain, le mieux plier aux disgrâces CIVILISÉEs, et le remplir de préventions contre l'étude de l'attraction et de la révélation permanente, où il eût pu trouver depuis 2500 ans l'issue de ses antiques malheurs. 


\section{DU LIBRE ARBITRE DE DIEU ET DE L’HOMME}

LES SYSTĖMES CIVILISÉS, toujours engagés dans l'un ou l'autre excès, prétendent, les uns, que la raison doit régner exclusivement, les autres que tout mouvement vient de Dieu; assertions de simplisme ; car si la raison triomphe et règne seule, comme sous Robespierre et autres hypocrites qui ne valent pas mieux, où sera l'emploi de Dieu qui intervient par attraction? D'autre part, si tout mouvement vient de Dieu, comment se fait-il qu'il existe des sociétés CIVILISÉES et BARBARES qui, étant purement coercitives et fondées sur la terreur des gibets, excluent évidemment pour les sept huitièmes de la population l'impulsion divine ou attraction, et remettent la régie du MOUVEMENT SOCIAL aux mains de la raison humaine à l'exclusion de Dieu ?

J'ai prouvé que Dieu est ennemi du despotisme et de l'exclusivité, et qu'en mouvement il laisse toujours moitié à faire aux créatures qu'il veut des associés et non des esclaves. Il veut laisser à notre industrie, à notre raison, l'honneur d'intervenir concurremment avec lui ; et pour preuve, abandonnez le mouton dans les forêts aux soins de la simple nature ou de Dieu seul, et il ne vous donnera plus les laines fines de Kaschmir et de Ségovie, sa toison deviendra rèche et grossière. Il faut donc que la raison, que l'industrie humaine intervienne de concert avec Dieu; mais nos sophistes, sous prétexte de tout céder à Dieu qui ne demande que moitié, lui ôtent réellement toute la moitié qu'il prétend, c'est-à-dire l'influence de l'attraction ou impulsion divine en concours avec la raison humaine, et non pas en conflit comme on le voit dans les ordres CIVILISÉ et BARBARE.

Il n'y a d'autre voie d'unité que de concilier les deux impulsions, celle de Dieu qui opère par attraction comme on le voit chez les astres et les animaux, et celle de l'homme qui doit opérer par raison ou science coïncidente avec l'attraction. 
Ce serait un problème bien embarrassant, si nous n'en tenions pas la solution ; elle est enfin donnée. On a vu que dans le mécanisme des SÉRIES PASSIONNELLES il y a toujours intervention libre de la raison humaine ou science du raffinement passionnel, et intervention libre de l'impulsion divine ou attraction.

Voilà donc le problème du libre arbitre pleinement résolu en sens composé, c'est-à-dire en concours des deux impulsions divine et humaine. Si l'on veut spéculer sur la solution en simple, sur l'exercice isolé de l'une des impulsions, l'on n'arrive qu'à l'oppression de Dieu et de l'homme. C'est ce que nous allons examiner.

Je m'appuie d'une comparaison qui fera sentir que si la philosophie est absurde en voulant donner à la raison le sceptre à l'exclusion de Dieu, la théologie ne l'est pas moins en ce que, sous prétexte de tout donner à Dieu, elle lui ôte réellement tout et ne laisse de libre arbitre ni à Dieu ni à l’homme.

Je suppose qu'un roi se plaise à faire habituellement une partie de cartes ou d'échecs avec son valet de chambre ; des courtisans persuadent à ce valet que le roi est mécontent de ne pas gagner et que lui valet court le risque d'être congédié et de perdre une excellente place. Dès ce moment, le valet ne s'étudiera plus qu'à laisser constamment la supériorité au roi, qui sera frustré, entravé par le fait ; car son intention n'est pas de terrifier le valet qu'il admet à jouer avec lui, mais de gagner une partie bien disputée, bien intriguée, et où la victoire soit flatteuse pour son amour-propre. Le roi assurément sera très mécontent lorsqu'il apprendra le secret de cette menée, et il pensera que gagner de la sorte, c'est ne plus gagner, ne plus jouer, et qu'en voulant lui donner tout l'avantage, on lui a réellement enlevé tout le charme de la lutte. Le valet, de son côté, aura de même perdu tout le plaisir du jeu par la persuasion qu'il faut céder au roi sur toutes les chances de gain ; et voilà deux individus frustrés tous deux dès l'instant où l'un veut tout céder à l'autre et mettre le despotisme, l'exclusivité, à la place de la balance et de l'équilibre.

Il eu est de même du libre arbitre. Si l'on ne donne pas moitié à Dieu et moitié à l'homme, sauf la tombée de mouvement qui doit être du côté de Dieu, il n’y a plus de liberté ni pour l'un ni pour l'autre, plus d'essor de l'attraction qui est le levier divin, plus d'exercice de 
levier humain qui est la raison convergente ou appliquée au RAFFINEMENT PASSIONNEL.

De là on conçoit que pour traiter la question du libre arbitre il faut extirper deux préjugés, le philosophique selon lequel on veut tout donner à l'homme ou à la raison, et le théologique par lequel on feint de donner tout à Dieu pour lui ôter effectivement tout.

Rappelons-nous que le mouvement est pour Dieu une fonction intriguée, composée, où il a besoin de laisser des chances aux créatures harmoniques, telles que planètes et hommes. Il ne laisse point ces chances aux animaux qui sont créatures simples, bornées à l'impulsion divine ou jeu de l'attraction et de l'instinct, sans concours de la raison ou raffinement passionnel ; aussi l'animal est-il stationnaire et n'avance-t-il pas au delà des limites de son instinct primitif. Les abeilles dans dix mille ans ne sauront pas mieux faire la ruche qu'elles ne la firent aux premiers âges du monde.

Il n'en est pas ainsi de l'homme, il est pourvu d'une raison progressive et alliable avec le levier divin ou attraction. Il est premier anneau de l'échelle composée, d'où il suit qu'il y a dans le mouvement des sociétés humaines deux leviers de mouvement à tenir en balance, et qu'en opprimant l'un des deux, soit le levier divin ou attraction, soit le levier humain ou raison, l'on n'aboutit qu'à les paralyser tons deux ; effet ridicule de nos sciences qui, voulant donner l'une tout à Dieu, et l'autre tout à l'homme, n'arrivent qu'à frustrer l'un et l'autre.

Que de nouvelles idées dans cette étude du mouvement social ! Que de préjugés bizarres chez ceux mêmes qui se vantent de les combattre ! Jugeons-en par la question qui nous occupe. Ici les théologiens sont les libéraux, car ce sont eux qui veulent donner tout à Dieu ; les philosophes sont les illibéraux, les despotes, voulant l'empire exclusif de la raison humaine ; et quand on envisage les résultats de leurs sciences - les trophées de la théologie et de l'inquisition sous Torquemada, les trophées de la philosophie et de la fraternité sous Robespierre —, doit-on s'étonner si l'humanité, confiée à ces deux guides présomptueux, marche comme l'écrevisse dans la carrière du bonheur, et si, après 3000 ans, elle est obligée d'en venir au précepte de Bacon : refaire l'entendement humain sur toutes les questions de mouvement social, et oublier tout ce que l'on a appris ! 
Beaux raisonneurs, qui voulez opérer tant de merveilles en balance, contre-poids, garantie, équilibre, et qui eu secret ne rêvez qu'usurpation et despotisme, apprenez en fait de balance et d'équilibre à ménager les droits respectifs. Vous avez cru, les uns que Dieu était tout, et l'homme rien ; les autres que Dieu n'était rien, et l'homme tout : c'est effet d'orgueil chez un parti et de bassesse chez l'autre. Pour vous élever aux théories d’ÉQUILIBRE, diminuez moitié de l'orgueil philosophique et moitié de la bassesse théologique; vous arriverez à concevoir que l'attraction doit entrer en balance avec la raison, que l'homme doit être associé et non valet de Dieu dans la régie du mouvement, sauf le pas honorifique ou tombée de balance qui appartient à Dieu.

Tant que déclinerez ce principe d'équilibre, cette balance et répartition d'influence entre Dieu et l'homme, vous serez inhabiles à concevoir les lois de l'équilibre passionnel qui est un jeu de mouvement dans lequel la raison humaine doit figurer, concourir avec l'action de Dieu. S'il nous laisse des chances nombreuses, entre autres celle des retards ou accélérations de découverte, celle de franchir plusieurs périodes et cumuler plusieurs créations, celle d'entraver par notre libre arbitre et abus de raison les opérations aromales de la planète, du tourbillon et de son univers ; si, dis-je, Dieu nous accorde cette infinité de chances, il est clair qu'il veut jouer avec la raison humaine une partie égalisée où les deux athlètes soient respectivement intrigués l'un par l'autre; où le Créateur comme la créature aient des moyens d'essor pour la CABALISTE, la PAPILLONNE et la COMPOSITE. Comment concevoir que Dieu soit juste et heureux s'il prive les créatures des moyens d'influencer le mouvement! Tout ne sera, je l'ai déjà dit, que despotisme, prédestination et fatalité. L’Être suprême, témoin des malheurs de notre globe, pourra donc se dire à lui-même : «C'est moi qui ai voulu la perpétuité de ces infâmes sociétés CIVIKISÉE, BARBARE et SAUVAGE, de ces raffinements de souffrance des hommes. » Tant de cruauté est-elle présumable dans la Divinité ? Mais si vous admettez qu'un Créateur juste et bon doive gémir de tant d'horreurs, n'est-ce pas admettre qu'il a inventé pour la régie des sociétés un ordre fortuné qu’il désirerait voir établi, et que son vœu est entravé par quelque erreur de la raison humaine, à qui il confie portion de la régie du mouvement ? Dieu créa les ressorts, les douze passions ; mais il laisse à l’homme le soin de les développer, en ménageant les 
droits respectifs de Dieu et de l'homme, en laissant égale part à l'attraction et à la raison. Tel devait être le sujet de nos études sur le mouvement social. Il fallait en raisonner comme des sciences, où nous faisons intervenir par moitié l'industrie humaine, mais pour féconder et non pour entraver la nature.

Quel rôle jouez-vous, philosophes et théologiens, dans cette étude de la destinée sociale et de la répartition de fonctions entre Dieu et l'homme? Vous, théologiens, figurez dans les rapports avec Dieu comme le pâtre qu'on fait asseoir à la table d'un grand seigneur, et qui dans sa stupeur ose à peine se placer sur le coin d'un fauteuil, perd contenance et présence d'esprit ; vous, philosophes, y figurez comme le démagogue Chabot, qui, introduit en députation chez Louis XVI, ne voulut pas ôter son chapeau devant le monarque. Excès pour excès, on ne saurait dire lequel est le plus ridicule : il faut partager la palme, et c'est le parti que je prendrai toujours dans les conflits entre la philosophie et la théologie.

Concluons et reconnaissons dans nos deux guides, rivaux d'impéritie, deux bambins scientifiques également ignorants sur la nature composée de Dieu et de l'homme, et sur le mécanisme composé de toute relation, libre arbitre ou autre, qui s'établit de l'homme à Dieu. Élevons-nous au-dessus des vues rétrécies de nos deux pédagogues : spéculons en sens composé, sachons concilier et faire marcher de front les droits de Dieu et de l'homme. Pour en assurer l'essor réciproque, est-il d'autre voie que les SÉRIES PASSIONNELLES, où l'attraction est toujours de concert avec la raison pour fonder l'équilibre des passions sur l'affluence des plaisirs?

Je ne me suis point arrêté aux subtilités de l'école sur le libre arbitre. Je ne les ai lues ni ne dois les lire, mon objet n'étant que de réclamer en faveur de Dieu, qu'on veut compter pour rien en libre arbitre et spolier et paralyser en comprimant les ressorts sociaux dont il est moteur. L'effet de cette violence est comparable à celui d'une partie où l'un des joueurs entrave son partenaire. Il ne reste plus d'intrigue et de charme ni pour l'un ni pour l'autre, et telle est la situation de Dieu dans ses rapports avec ce globe où nos sciences l'ont forcé à abandonner la direction active du mouvement. Il n'intervient que passivement, par l'entretien des ressorts passionnels que l'homme ne peut pas détruire, mais que Dieu ne peut pas diriger en harmonie tant qu'on ne lui 
accorde pas sa moitié de régie, sa garantie de libre concours avec la raison humaine, concours qui ne peut s'établir que par la synthèse de l'attraction ou mécanisme des SÉRIES PASSIONNELLES.

OBJECTION : "Si Dieu est assez généreux pour concéder moitié à la raison humaine dans la direction sociale des globes, il ne devrait pas lui concéder tout, comme il paraît le faire, en accordant à la raison cette faculté d'éliminer Dieu de la direction active ; il aurait dû se ménager des moyens d'amener la raison à résipiscence. »

Un tel argument rentre dans le système du fatalisme. La raison humaine y serait assujettie, si elle ne jouissait pas de la plénitude d'option entre le concert avec Dieu ou l'isolement de Dieu. Elle est toujours à temps de revenir à cette alliance, et les égarements de raison dans un siècle n'interdisent pas au siècle suivant le retour à des opinions plus sensées. Ainsi Dieu, dans cette exclusion, conserve des chances d'intrigue nécessaires à sa $10^{\mathrm{e}}$ passion, la CABALISTE; il conserve les alternats nécessaires à sa $11^{\mathrm{e}}$ passion ou PAPILLONNE; elle serait entravée si tous les globes marchaient d'un même pas à l'harmonie, ou restaient à perpétuité en subversion, quand l'esprit philosophique les y a engagés. Enfin Dieu conserve les chances de COMPOSITE nécessaires à sa $12^{\mathrm{e}}$ passion, car sur cette masse d'environ $1 / 8^{\mathrm{e}}$ des globes qui dévient de la bonne voie et tombent dans l'arbitraire philosophique, il en est toujours bon nombre qui reviennent successivement à l'équilibre. Quant aux autres, les voies de retour ne leur étant pas fermées et le progrès du luxe les obligeant à chercher de plus en plus une issue des misères de la limbe sociale, tout est disposé de manière à intriguer le mouvement au suprême degré pour Dieu et pour l'homme, sans jamais anéantir le libre arbitre de l'un et de l'autre, malgré les entraves temporaires qu'il peut éprouver et qui tournent toutes au préjudice de l'oppresseur.

En effet, qui est-ce qui porte la peine de cette entrave ? Elle pèse légèrement sur Dieu, et violemment sur l'homme qui la cause. Dieu n'éprouve d'autre contre-temps que d'abandonner la partie sur un globe, sur un tourbillon, sur un univers qui diffèrent à admettre son intervention, admise avec joie par des milliards d'autres univers ; mais l'homme est lésé sept fois plus, en ce que l'entrave, qui n'est que négative pour Dieu, devient positive pour l'homme. Celui-ci n'a pas la faculté de s'isoler de la gestion d'un monde social mal géré ; il est ré- 
duit à y souffrir, et cette peine frappe sur les $7 / 8^{\mathrm{e}}$ des hommes, car on en trouve à peine $1 / 8^{\mathrm{e}}$ d'heureux. Nous travaillons donc à notre supplice par le refus d'admettre Dieu pour moitié dans la régie du mouvement. Il est assez dédommagé de nos refus par l'adhésion de tant de milliards de globes et d'univers, qui font leurs délices de vivre sous son code social et de partager avec lui le sceptre du mouvement dont il a l'insigne bonté d'offrir moitié à la raison humaine, toujours admise à accepter ce partage, dès qu'elle sera lasse des désastres où elle se plonge en voulant gouverner seule sous la tutelle des philosophes et des théologiens, dont les dogmes, sous diverses formes, ne sont toujours que la sagesse humaine excluant et opprimant Dieu et l'attraction. 


\section{DU LIBRE ARBITRE DE L'HOMME, EN SIMPLE ET EN COMPOSÉ ; EN POSITIF ET EN NÉGATIF}

EN NOUS DÉPEIGNANT Dieu comme un maître terrible, étayé de démons, de brasiers et de serpents, on nous a donné de lui des notions si fausses que les esprits répugnent généralement à spéculer sur la bonté de Dieu, sur ses intentions généreuses et libérales, telles que je viens de les décrire : aussi le siècle s'est-il jeté dans l'irréligion par dédain pour les enfers et serpents dont on arme la Divinité. Il en est resté de fâcheuses traces, un penchant à isoler Dieu de toute coopération sociétaire avec l'homme. De là vient que des lecteurs ne goûteront pas la thèse du libre arbitre présentée en dépendance réciproque de Dieu et de l'homme, et partage d'intervention comme je viens de le faire ; il convient peut-être mieux de traiter le sujet humainement, sans faire mention du concours de la Divinité. Souvent une question gagne à être envisagée sous plusieurs faces, et nous pouvons restreindre celle-ci à l'homme seul.

Dans cette hypothèse, le libre arbitre se divisera en SIMPLE et en COMPOSÉ : simple, s'il n'opte que pour la raison seule ou la passion seule ; composé, s'il opte pour toutes deux, s'il parvient à une raison coïncidente avec l'attraction passionnée.

Quelques sybarites riches, heureux et prudents, peuvent se donner ce plaisir de raison et attraction coïncidentes. On voit de ces hommes tout occupés à calculer leurs jouissances par poids et mesures, habiles à se ménager une série judicieuse de plaisirs et assez exercés pour parer aux astuces et aux vides de la société. Ils se prouvent à eux-mêmes et aux autres qu'ils sont des prodiges la raison, mais d'une raison positive ou épicurienne, qui s'accorde avec le jeu des passions et n'intervient que pour les seconder et raffiner. C'est une raison composée ou combinée avec l'attraction : c'est enfin le but de la nature, but que ne remplissent point la philosophie et la théologie, qui conseillent 
d'aimer les privations parce qu'elles ne savent pas nous donner les richesses. Elles nous conseillent donc une raison NÉGATIVE et SIMPLE, ou raison ISOLÉE d'attraction.

On trouve la sagesse positive chez quelques heureux du siècle, dans le cas où ils possèdent santé, richesse et prudence en pays de pleine paix et abondance. A défaut de la santé et de la richesse, ils pourraient avoir une raison stoïcienne ou compatible avec les privations : ce serait une raison simple divergente et non pas convergente avec les passions. Elle ne serait plus un libre arbitre composé, mais une scission raisonnée de la raison avec l'attraction, enfin un état moral négatif et simple, triste lot que nous alloue la philosophie sans nous laisser de liberté sur le choix du mieux, qu'on n'obtient que par la richesse.

Quant à l'épicurisme raisonné, qui est vraiment un libre arbitre composé, il est trop certain que la multitude n'en jouit pas. Elle n'arrive pas même à l'état moral ou liberté simple divergente. J'excepte quelques vieillards opulents, pour qui les glaces de l'âge transforment en plaisir une modération raisonnée. Quant aux jeunes gens que la fougue de l'âge ne pousse qu'aux excès, ils ne tardent pas à payer cher l'usage du libre arbitre simple, et je dis simple chez eux, puisqu'il est essor d'attraction sans concours de raison.

Ceci n’a trait qu'à la classe riche. On voit qu'elle a quelques lueurs de libre arbitre composé, et qu'elle ne jouit du simple que pour son malheur, puisque la dominance de raison n'est qu'un pis-aller de la vieillesse et la dominance d'attraction qu'un écueil pour la jeunesse.

De là naissent des dogmes de circonstances au sujet du libre arbitre. On ne veut pas le contester à l'homme : ce serait tomber dans les préjugés de fatalisme, confondre le crime avec la vertu, et faire passer sur Dieu la responsabilité des torts de l'homme. Pour éviter ces écarts, la science a recours aux libertés négatives. A force de sophismes, elle transforme en plaisirs nos privations et nos servitudes : elle nous apprend que le libre arbitre doit nous conduire à faire ce qui nous déplaît et ne pas faire ce qui nous plaît. La philosophie et la théologie nous prouvent que le vrai républicain et le vrai chrétien doivent être en guerre avec leurs passions, être les bourreaux d'eux-mêmes pour agir selon Dieu et selon la raison. 
La théologie oublie que selon ce dogme, Dieu, à titre de distributeur de l'attraction, tomberait dans le double vice d'impéritie et de persécution, s’il distribuait aux créatures des penchants incompatibles avec leur libre arbitre attractionnel. Quant à la philosophie, elle oublie qu'on lui demande les libertés positives, et non les négatives qu'elle décore du nom de raison, classant au rang de mal toutes ou presque toutes les jouissances positives : en quoi elle est bien secondée par la théologie.

On pourrait là-dessus inviter ces deux sciences à se concilier sur les définitions du bien et du mal. On leur observe que ce qui est bien dans un siècle ou dans un pays a été mal dans un autre siècle et dans un autre pays, en dépit des principes qu'on dit éternels. Il est prouvé que l'anthropophagie, l'adultère, le suicide, l'inceste, ont été vertus et le sont encore dans divers pays. Il n'y a pas si longtemps que c'était une vertu en France de dénoncer son père et de l'envoyer à l'échafaud. Il faudrait donc, avant de raisonner sur le bien et le mal, que nos savants parvinssent à s'accorder en définitions. Ils en sont plus loin que jamais. Un siècle qui se vante de chercher la vérité et qui prône le commerce simple ou libre exercice du mensonge, un tel siècle est-il admissible à opiner sur ce qui est bien ou mal, quand par ce fait il érige en bien la pratique du mensonge et de toutes les astuces ?

Passons-leur cette absurdité, et admettons que le bien et le mal soient des connaissances fixes comme la géométrie ; il restera à demander pourquoi, si nous avons le libre arbitre, nous sommes punis par Dieu et les hommes quand nous optons pour le mal moral, plus attrayant que le bien moral. Nous nous trouvons donc dans le cas d'un affamé à qui l'on dirait : " Je te permets de manger ce pain, mais si tu en manges une miette, je te brûle la cervelle. » Celui qui traiterait de la sorte un malheureux pressé par la faim, pourrait-il se vanter de lui avoir donné le libre arbitre ? Loin de là, il n'aurait fait qu'ajouter la contrainte au besoin, et doubler la souffrance par l'aspect du bien désiré.

Telle est, dans l'ordre CIVILISÉ et BARBARE, la situation des $7 / 8^{\mathrm{e}}$ des hommes. La religion dont on leur conseille l'usage n'est pour eux qu'un raffinement de supplice, qu'une contrainte ajoutée au besoin ; et le libre arbitre, en cas qu'on veuille l'admettre, n'est qu'un garant de supplice en ce monde et en l'autre, car en cédant sans raisonner à 
l'impulsion naturelle ou attrayante, nous serons suppliciés ; et en cédant à la raison qui nous ordonne de résister à nos passions, nous éprouverons double tourment, contrainte et besoin.

Cherchons hors de nos sociétés quelques notions plus recevables sur le libre arbitre.

Un chef sauvage à qui le roi d'Angleterre adressait cette question : "Vos sujets vous obéissent-ils bien ? « répondit : " Pourquoi non ? Je leur obéis bien moi-même. » Ce chef, dans sa réponse mille fois plus sage que le bel esprit de nos moralistes, nous donne la solution du problème du libre arbitre. Il doit être réciproque, et ne peut exister qu'autant que les deux éléments passion et raison sont en convergence ; qu'autant que les inférieurs ont le droit de désobéir dans le cas où le commandement du supérieur irait contre leur vœu : c'est donc au supérieur à ne commander que ce qui leur plaît collectivement et individuellement. S’il observe cette règle, il sera obéi ; s’il n’est pas obéi, c'est qu'il aura enfreint la condition de satisfaire la volonté collective et individuelle.

Je sais combien de tels principes sont inadmissibles en CIVILISATION : aussi ai-je dit qu'il fallait chercher hors de cette société pour découvrir un emploi collectif du libre arbitre.

Du moment où l'on met en jeu la contrainte, les châtiments, pour faire exécuter un ordre, il ne remplit aucune des conditions du libre arbitre, et si l'on veut nous persuader que Dieu ou la loi nous donne cette prérogative, il faut d'abord supprimer les enfers, les gibets et autres voies coercitives, borner les ressorts du mouvement aux deux éléments indiqués, savoir : l'impulsion directe ou adhésion irréfléchie, qu'on nomme attraction, et l'impulsion indirecte ou adhésion réfléchie, qu'on nomme raison positive, convenance de plaisir calculé.

On objectera que les CIVILISÉs, abrutis par la pauvreté, n’ont aucun discernement en affaires sociales, et que la raison consiste à les violenter pour leur propre bien. Je le sais ; et j'ai observé, en parlant des jantes larges, de l'unité monétaire, etc., qu'on ne connaît pas encore l'emploi régulier des contraintes utiles ; mais, d'après ce principe, les CIVILISÉs n'ont donc pas de libre arbitre ou tout au moins ils ne l'ont qu'en négatif, ils n'ont que l'option du moindre mal: souffrir l'indigence et la faim pour éviter les gibets et les enfers. 
Le problème étant d'arriver au libre arbitre POSITIF et COMPOSÉ, il est clair que la CIVILISATION ne peut nous le donner et nous ne l'obtiendrons que par l'ISSUE DE CIVILISATION et l'entrée dans un nouvel ordre social qui, assurant au peuple l'aisance, le luxe, les plaisirs, et par suite le goût du bon ordre, l'attachement au régime de l'industrie attrayante, dispensera de recourir à la contrainte.

Si les voies coercitives conduisaient la multitude à son but, au bonheur composé ou destination de l'homme, telle qu'elle est définie dans le prologue, on pourrait approuver les deux sciences philosophique et théologique d'avoir comprimé le libre arbitre et l'avoir, à force de sophismes, restreint à l'essor négatif : mais au lieu de bonheur composé elles conduisent l'immense majorité au malheur composé, résultat constant des sociétés CIVILISÉE et BARBARE, et ne nous assurent pas même l'exercice de la raison, l'un des deux éléments du libre arbitre. Elles ne peuvent pas s'entendre sur la fonction de cette raison dont elles modifient sans cesse les oracles dans leurs innombrables codes et systèmes. Leur contradiction est pire encore quant aux résultats, puisque ces codes nous donnent tout le contraire de ce que la raison philosophique nous avait promis, n'opèrent partout que la permanence des fléaux.

C'est donc à bon droit que l'homme se plaint d'être plus malheureux que les animaux qui, étant destinés à l'attraction simple ou passion pure, jouissent du plein essor et du libre arbitre ; tandis que nous, destinés au libre arbitre composé ou essor combiné de la passion et de la raison, nous ne jouissons ni de l'un ni de l'autre. Quelques exceptions, bornées au plus à $1 / 8^{\mathrm{e}}$ des hommes, ne servent qu'à constater l'exclusion générale.

Ces considérations n’ont pas échappé aux savants, qui ont escobardé autant que possible sur ce problème du libre arbitre. Quelques-uns pourtant, et notamment Voltaire, ont fait sur cet asservissement des hommes et sur les vues de Dieu relativement au bien et au mal, des arguments très forts et auxquels la science CIVILISÉE ne saurait répondre.

L'embarras des savants naît de ce qu'ils n'envisagent le mouvement qu'en sens rétrograde. Le voyant parvenu à la $4^{\mathrm{e}}$ limbe ou CIVILISATION, ils en concluent qu'il ne peut pas s'élever plus haut et 
ne spéculent que sur la carrière déjà connue. C’est raisonner comme celui qui aurait dit, avant l'expédition de Colomb : «J'ai fait mille lieues dans l'Atlantique, je me suis avancé plus loin qu'aucun autre navigateur. Je n'ai pas découvert de nouveau continent : donc il n'en existe pas. » Chacun aujourd'hui saurait lui répondre : "Mille lieues n’ont pas suffi ; retournez et faites-en deux mille, trois mille au besoin. » Tel est le tort des CIVILISÉs : ils ne trouveront rien de satisfaisant sur le problème du libre arbitre ni sur toutes les questions du mouvement social, tant qu'ils voudront se borner aux échelons connus, aux quatre limbes SAUVAGERIE, PATRIARCAT, BARBARIE, CIVILISATION.

Nous allons, en spéculant sur la continuation de l'échelle, réfuter leurs sophismes contre le libre arbitre composé ou libre exercice de la passion et de la raison combinées.

Divisons l'examen en rétrograde et en extrograde : nous comprendrons dans la partie rétrograde l'état présent et passé du mouvement social, et dans l'extrograde l'état futur, les sociétés à venir. 


\section{DU LIBRE ARBITRE EN CARRIÈRE RÉTROGRADE}

TANT QUE nous sommes en limbes obscures, dans les cinq périodes SAUVAGE, PATRIARCALE, BARBARE, CIVILISÉE, et même en GARANTISTE, nos passions nous poussent plus ou moins au mal. Or, si l'intention de Dieu est que nous ne commettions pas le mal et que nous soyons réprimés, contenus par le frein des lois et de la religion, il semble avoir voulu notre asservissement, et nous paraissons fondés à lui reprocher un état de choses qui ne nous laisse pas autant de liberté qu'aux animaux en qui l'essor libre de la passion n'est point un vice, et qui jouissent vraiment du libre arbitre simple, ou passion sans raison. Pourquoi l'espèce humaine, qui est leur supérieur, n'a-t-elle pas comme eux le droit de se livrer à ses passions, et pourquoi Dieu lui en a-t-il donné d'assez vicieuses pour qu'il soit nécessaire de la priver du libre arbitre ? En nous donnant pour ressorts sociaux la passion et la raison, pourquoi ne nous a-t-il pas donné la raison en dose proportionnée à la passion, mais au contraire en quantité si faible qu'elle équivaut à zéro chez la multitude et même chez les savants qui, parlant sans cesse de raison et de modération, n'en ont pas l'ombre dès que leur passion est émoustillée ?

Dieu a-t-il donc voulu nous priver du libre arbitre en nous privant à peu près de l'un des deux éléments qui doivent y concourir, et donnant à l'autre, à la passion ou attraction, une intensité désordonnée qui oblige à la tenir en compression perpétuelle chez les peuples INDUSTRIEUX, CIVILISÉS, et BARBARES, et non chez les SAUVAGES

Ainsi, lorsqu'on se borne, en mouvement social, à envisager la carrière rétrograde, le présent et le passé, on est induit à penser, ou que Dieu ne veut pas le libre arbitre dans les sociétés INDUSTRIELLES, ou que Dieu ne veut pas l'industrie s'il veut l'exercice du libre arbitre. 
De ces deux opinions, la deuxième est inadmissible, car l'abandon de l'industrie, en nous ramenant à l'état sauvage, rend bien le libre arbitre aux hommes qui sont réellement libres en SAUVAGERIE, mais non pas aux femmes qui forment moitié de la population et sont très comprimées chez les SAUVAGEs; et d'ailleurs les hommes n'y jouissent que de la liberté politique et non de la liberté passionnelle. Quelques SAUVAGES ont des lois très rigoureuses contre l'adultère et autres essors de passion, qu'ils punissent par des supplices légaux chez l'homme ainsi que chez la femme.

On ne peut donc pas conclure que Dieu ait voulu fonder le libre arbitre sur l'abandon de l'industrie, car les hommes SAUVAGES sont entravés sur plusieurs passions, et leurs femmes le sont presque sur toutes.

Il reste à disserter sur la première proposition : Dieu semble interdire le libre arbitre aux sociétés INDUSTRIELLES, puisque la compression des $7 / 8^{\mathrm{e}}$ de leurs membres est nécessaire, et que le $1 / 8^{\mathrm{e}}$ privilégié, qui comprime les $7 / 8^{\mathrm{e}}$, est encore entravé lui-même dans une foule de passions, les grands n'ayant pas, à beaucoup près, le libre essor des leurs, et s'en plaignant amèrement jusque sur les trônes.

C’est ici que nous allons sentir l'inconvénient de ne spéculer que sur les âges rétrogrades ou CARRIÈRE DE LIMBE, dont l'analyse mène à conclure que Dieu ne veut de libre arbitre ni pour les sociétés INERTES ou SAUVAGES qui n'en jouissent pas, ni pour les sociétés industrielles qui en jouissent encore moins. Que nos sciences deviennent ineptes quand on les attaque sur ce résultat! Elles sont en libre arbitre ce qu'elles sont sur tous les points: la perfectibilité en paroles et l'absurdité en action ; mais au risque de redites nécessaires il faut retracer leurs côtés faibles sur ce sujet.

Nos théologiens et philosophes n'ayant découvert aucun moyen de concilier les deux impulsions élémentaires du libre arbitre, savoir : l'attraction qu'on nomme le mal, et la sagesse qu'on nomme le bien, nous réduisent au libre arbitre simple, à l'option pour l'un des deux éléments, option qui constitue l'homme en état de guerre permanente avec Dieu et avec lui-même :

1. Avec Dieu, puisque l'attraction vient de Dieu, et que la sagesse venant de l'homme veut exclure l'attraction ou impulsion divine, en 
comprimer au moins les $7 / 8^{\mathrm{e}}$, ce qui est comprimer le tout en thèse de mouvement ;

2. Avec lui-même, puisque la sagesse qui vient de l'homme est un océan de contradictions, d'où l'on voit naître, dans une seule ville, à une seule époque, 278 opinions divergentes sur une seule question.

Ainsi, en proscrivant l'attraction, nous tombons sous la tutelle d'une prétendue sagesse fort éloignée du libre arbitre, car elle ne peut s'accorder ni avec Dieu, ni avec l'homme, et n'admet l'impulsion ni de Dieu, ni de l'homme. Jugeons-en par une courte analyse de ses deux branches, théologie et philosophie: l'une en guerre avec l'attraction, l'autre en guerre avec la raison et l'attraction.

La théologie, qui nous défend l'usage de la raison par le précepte de fide est (c'est article de foi), nous interdit aussi l'obéissance à l'attraction. Ainsi, sous prétexte de donner tout à Dieu, elle ne laisse rien ni à Dieu, ni à l'homme. Cependant elle prétend venir de Dieu : mais comme il y a des théologiens par centaines et que chacun a produit et produit encore des sectes rivales qui se damnent réciproquement, on en doit conclure que si tontes les religions CIVILISÉES, BARBARES et SAUVAGES viennent de Dieu, l'esprit de Dieu n'est qu'incertitude et contradiction, et que Dieu ne veut pas le libre arbitre de l'homme, puisque, dans les $9 / 10^{\mathrm{e}}$ de ces religions, il dévoue les mécréants aux brasiers éternels ; ce qui n’est plus leur laisser libre option, liberté de jugement.

La philosophie, qui dénonce le système oppressif de sa rivale, estelle moins intolérante ? N'a-t-elle pas aussi ses articles de foi ? Ses risibles assertions sur l'existence de la liberté, là où l'homme n'a pas le droit de manger quand la faim le presse ? En voulant étouffer l'attraction ou impulsion divine, elle la remplace par une raison qui sanctionne tous les actes de tyrannie, pourvu qu'ils soient fardés de beaux verbiages. L'une fait au nom de la raison ce que l'autre fait au nom de Dieu. Toutes deux approuvent qu'on envoie au supplice le malheureux qui, pressé par la faim, dérobe un pain dont l'attraction, écho de Dieu, lui fait une loi de se nourrir.

Voilà donc la science divisée en deux sectes primordiales qui, sous divers masques, ne veulent reconnaître ni Dieu ni la raison dont elle se 
disent les interprètes, et punissent en pratique ce libre arbitre qu'elles promettent en théorie.

D'où naît ce conflit d'absurdités ? De ce que chacune des deux spécule sur le simple qui n'est pas applicable à l'homme (sinon en relais de composé). Chacune s'attache à une prétendue sagesse divine ou humaine, qui toujours exclut l'attraction et borne le libre arbitre à un seul de ses deux éléments. C'est l'anéantir, car en principe de mouvement "Tout mécanisme essentiellement composé, qu'on veut réduire au simple, n'arrive point au simple, mais tombe en composé subversif ou conflit de ses deux éléments. " C’est une règle qui nous expliquera toutes les absurdités CIVILISÉES, dont je renvoie l'examen à la touche du duplicisme (PIVOTALE MINEURE).

C'est assez prouver que pour arriver au libre arbitre composé et surtout positif, ou liberté d'option sur les jouissances, le problème est de développer combinément l'attraction et la sagesse ou raison ; et, pour y réussir, il n'est d'autre voie que de déterminer un nouvel ordre social où les deux ressorts soient compatibles. C'est sur quoi n'ont pas voulu spéculer les deux sectes qui se disputent le sceptre de l'opinion. En conséquence elles ont mis en scène le libre arbitre simple ou option pour l'un des deux ressorts, pour la raison philosophique ou religieuse, à l'exclusion de l'impulsion divine. De là naît le cercle vicieux que je viens d'analyser, et qui ne laisse à l'homme aucune liberté, à Dieu aucune influence directe sur l'ordre social.

Pour démontrer que Dieu désire nous assurer le libre arbitre composé, il faut raisonner sur la carrière extrograde, sur les périodes sociales plus élevées en échelle que la CIVILISATION et autres limbes obscures. 


\section{DU LIBRE ARBITRE EN CARRIÈRE EXTROGRADE}

CE N'EST PAS au début de l'ouvrage que j'aurais pu traiter un problème aussi inabordable pour des CIVILISÉs que celui du libre arbitre composé et positif. Il fallait préalablement dépeindre le nouvel ordre de choses où ce bienfait peut être accordé à l'homme, cet état d'harmonie passionnelle où, les plaisirs étant innombrables et les travaux métamorphosés en plaisirs par les rivalités et appâts industriels des SÉRIES, le plus pauvre des hommes jouit à chaque instant d'une option de plaisirs.

Dans leur distribution et graduation, il a besoin d'un exercice continuel de sa raison, afin de pouvoir aborder à la masse de jouissances, et les rehausser l'une par l'autre au moyen d'un judicieux enchaînement. On vient de lire les tableaux de cet ordre, et l'on peut juger maintenant combien il est impossible, en spéculant sur la CIVILISATION, de concevoir aucun essor combiné des deux éléments du libre arbitre, attraction et raison, qui, dans ce nouvel ordre, se prêtent un appui mutuel, au lieu de se contrecarrer comme aujourd'hui par effet de la pauvreté qui limite les jouissances à $1 / 8^{\mathrm{e}}$ du corps social.

Tant qu'on ignore cette destinée heureuse que Dieu réserve à l'homme, on ne peut, je l'ai déjà dit, spéculer que sur un libre arbitre négatif et simple, c'est-à-dire une option pour le moindre mal et pour l'un des deux éléments, favorisé an détriment de l'autre. Tel est notre sort en CIVILISATION.

Mais si l'on vent envisager le mouvement en sens extrograde, s'élever au delà des sociétés connues, raisonner sur le mécanisme des SÉRIES PASSIONNELLES, tous les problèmes de bonheur social deviennent des jeux d'enfants, et rien n'est plus facile que de développer en 
plein accord l'attraction et la raison, s'élever ainsi à la jouissance du libre arbitre composé et positif.

Jusque-là toutes les subtilités de l'école ne sauraient répondre aux arguments sur le malheur de l'homme, sur la malfaisance apparente de Dieu et sa préférence pour les animaux, que nous voyons pourvus d'un bonheur suffisant, selon leur libre arbitre, tandis que le monde CIVILISÉ ne cesse de gémir sur l'étendue de ses malheurs.

Destiné à l'équilibre composé, au libre arbitre composé, l'homme depuis 3000 ans ne s'étudie qu'à chercher des lois d'équilibre simple, qu'à restreindre ses prétentions, au lieu de s'essayer dans un champ plus vaste que cette CIVILISATION qui réduit le mécanisme domestique au-dessous même du minimum : car, en supposant que l'homme soit destiné à vivre par familles, au moins faudrait-il, selon la nature et le libre arbitre, laisser à cette famille toute l'extension possible en admettant tontes ses branches. Notre système, au contraire, la limite au couple conjugal et privilégié. On peut le défier d'atteindre à une plus forte réduction ; et des hommes qui fondent ainsi leur mécanisme social sur l'entrave absolue des passions, veulent disserter sur le libre arbitre, nous prouver son existence !

Il règne dans les SÉRIES PASSIONNELLES, parce que l'abondance de plaisirs concurremment offerts suffit à modérer la passion, déjà calmée par une heure de jouissance. Il y a, dans un tel ordre, alternat de la passion et de la raison, balance et contre-poids de l'impulsion divine ou attraction qui règne dans le cours des séances, et de l'impulsion humaine on raison qui en gradue la distribution de manière à prévenir les excès par la rapide succession et le contraste judicieux des plaisirs. C'est le seul moyen de mettre sans danger l'attraction aux prises avec la raison.

S'il y a rareté de plaisirs, l'attraction, trop longtemps privée, se change en fougue déraisonnable, et de là vient que, parmi nous, l'excès règne non seulement dans les séances de plaisir, mais dans les distributions de plus longs termes qui s'étendent aux âges extrêmes. Aussi voit-on beaucoup de gens qui, à soixante ans, veulent mener le train de vie qui ne convient qu'à trente : excès inhérent à la rareté des plaisirs CIVILISÉs, qui ne sont jamais répartis de manière à satisfaire l'imagination en temps et lieu convenables. 
Quand la balance et l'option seront régulièrement établies sur une période quelconque, séance, journée, semaine, mois, année ou phase de la vie, il y aura libre arbitre dans l'impulsion divine ou directe, qui est l'attraction, et dans l'impulsion humaine ou indirecte, qui est la raison. Celle-ci n'aura plus la tâche impossible de modérer l'attraction, mais seulement de l'éclairer et de la diriger dans les options et graduations de l'affluence de plaisirs qui sera offerte. La raison alors sera écoutée, parce qu'elle servira et raffinera l'attraction. Aujourd'hui qu'elle essaie de l'entraver elle est foulée aux pieds ; il n’y a donc entre elles d'autre moyen de concert que le mécanisme des SÉRIES, qui les rend nécessaires l'une à l'autre dans la répartition des plaisirs.

Dans ce nouvel ordre, il y aura une légère supériorité du côté de l'impulsion divine, en ce que l'attraction aura l'initiative. Les options et décisions de la raison ne seront qu'une capitulation avec l'attraction, mais enfin ce sera un exercice de la raison humaine, en concurrence avec l'impulsion divine, pour raffiner nos plaisirs et nous laisser l'honneur de partager avec Dieu la direction de notre individu et de nos sociétés.

Et dans ce régime d'où naîtra tant de bonheur, n'est-il pas juste que la balance penche pour l'autorité divine ou attraction ? Et puisque dans tout mouvement il faut une tombée de balance, qui existe déjà parmi nous dans les proportions physiques et numériques des sexes, peut-on douter que dans nos relations avec Dieu, dans la direction du mouvement social, la tombée ne doive, pour notre bien même, appartenir an Suprême Pondérateur, avec qui il nous semblera si doux de partager la régie sociale, quand nous connaîtrons le sort heureux qu'il nous a préparé ! Mais Dieu, aussi généreux que nous sommes imprudents, ne veut ni d'une confiance aveugle ni d'une autorité absolue, et il nous a ménagé dans les SÉRIES PASSIONNELLES un partage réel d'autorité, une entremise permanente de la raison concurremment avec l'attraction. Il ne veut, dans cet ordre équilibré, que le faible surplus de tombée nécessaire à assurer notre bonheur, qui serait nécessairement compromis du moment où la raison humaine, sujette à l'erreur, viendrait à surpasser en influence l'impulsion divine on attraction.

On peut maintenant apprécier la justesse des plaintes élevées sur le parallèle de l'homme et de l'animal dont nous déplorions à bon droit 
de ne pas égaler le bonheur, l'exercice du libre arbitre. Il restait à savoir que notre bonheur doit être double du sien, savoir : libre exercice d'attraction et de raison coïncidentes. Nos sages, pour avoir voulu les faire agir en divergence et nous borner à jouir d'une seule, nous ont ôté l'exercice de l'une et de l'autre, en les armant l'une contre l'autre : effet nécessaire du mouvement simple qui, appliqué à l'homme, produit toujours la DUPLICITÉ D’ACTION parce que, les ressorts élémentaires des passions humaines étant composés en tout sens, dès qu'on veut n'en faire jouer qu'un seul à l'exclusion de l'autre on établit le conflit de tous deux ; et c'est l'erreur perpétuelle de nos prétendus sages, toujours aheurtés à spéculer sur l'essor simple et la simple nature, qui est attribut de l'animal et non pas de l'homme.

Récapitulons. Sons le rapport de l'unité : quelle unité trouveronsnous dans les deux méthodes simples que nous proposent la philosophie et la théologie ? Méthodes identiques au fond, malgré le contraste de moyens, car elles ne sont, sous diverses formes, qu'une répression de celui des deux ressorts qui vient de Dieu.

La philosophie veut limiter le libre arbitre au ressort purement humain, à l'emploi de la raison. C'est une scission manifeste avec Dieu : c'est l'exclure d'intervention et sans pour cela faire régner la raison ni le libre arbitre, dont on ne voit aucune trace en mécanisme cIVILISÉ.

L'autre science, la théologie, met en scène des révélations divines qui nous condamnent à souffrir, par raison, des privations en ce monde. C'est mettre Dieu en scission avec lui-même, puisqu'il est créateur de l'attraction qui nous stimule incessamment. Ce sont deux systèmes schismatiques avec Dieu : l'un repousse l'intervention divine en déclinant les droits de l'attraction qu'il veut soumettre à la morale répressive, l'autre ne fait intervenir la Divinité qu'en sens absurde et outrageant pour elle : car il la suppose armée contre son propre ouvrage, s'efforçant de comprimer l'attraction qu'elle a créée, et voulant que l'homme se ligue avec Dieu pour opprimer cette impulsion dont Dieu protège l'essor chez les autres créatures. Supposer à Dieu ces vues insensées dans sa régie du mouvement social, n'est-ce pas être en pleine scission avec lui ?

Et si l'on considère que ces deux méthodes anti-unitaires n'aboutissent qu'à entretenir les 7 fléaux (INDIGENCE, FOURBERIE, 
OPPRESSION, CARNAGE, INTEMPÉRIES OUTRÉES, MALADIES PROVOQUÉES, CERCLE VICIEUX), et nous priver en tout point du libre arbitre, quel sens faut-il attacher aux verbiages d'unité dont s’affublent les deux sciences, et comment douter que dans une théorie d'unité réelle, il ne faille s’isoler de ces deux sentiers d'erreurs, et spéculer sur le libre arbitre comme sur tous les ressorts du mouvement passionnel, en emploi composé convergent des deux éléments, l'attraction et la raison?

Quelle réplique opposer à ce principe, maintenant que la découverte du calcul synthétique de l'attraction démontre qu'il ne peut exister hors des SÉRIES PASSIONNELLES ni règne de la raison, ni essor de l'impulsion divine ou attraction, ni libre arbitre de l'homme, ni unité de l'homme avec Dieu et le système de l'univers !

Du reste, à quoi bon s'appesantir sur ces prétentions de libre arbitre dans notre CIVILISATION, où l'homme n'a pas la liberté de manger quand il souffre de la faim et qu'il se voit entouré d'une affluence de comestibles étalés pour la provoquer ? Un dogme qui se rit à ce point de l'évidence mérite-t-il les honneurs de la réfutation ? Et qu'y avait-il à dire jusqu'à présent sur le libre arbitre, sinon de s'étonner de la contradiction apparente de Dieu, qui, accordant ce bienfait aux astres et aux insectes, le refuse à l'homme seul ? Voilà l'énigme expliquée par le parallèle du libre arbitre simple que l'on veut inutilement introduire en CIVILISATION, et du composé, qui n'est applicable qu'à l'HARMONIE. On a vu combien elle est facile à organiser, et combien cette fondation s'accordera avec le libre arbitre unitaire. L'issue de l'abîme nous est ouverte, hâtons-nous d'en sortir, et, pour terminer par un dictum moral, " ne perdons pas à discuter sur le libre arbitre les moments destinés à en jouir. » 


\section{CLASSEMENT DES LIBERTÉS VRAIES ET ILLUSOIRES}

Ne CRAIGNONS PAS de multiplier les tables. Sans cette précaution, il ne reste souvent d'une lecture que des notions confuses. Une table vient à propos à la suite des débats, pour classer et graver dans la mémoire divers détails dont les lectures n'ont laissé que de vagues souvenirs.

Nous n'avons que quatre modes à envisager dans l'essor des libertés. Leur classement bien précis mettra fin à toutes les controverses politiques et théologiques sur les libertés. Le problème se borne à décider lequel des quatre modes assure le plein bonheur, et il n’y aura pas à hésiter sur la décision.

TABLE DES QUATRE EXERCICES DE LIBERTÉ

\begin{tabular}{|l|l|l|}
\hline La composée positive ; & convergente directe : & 2 éléments. \\
\hline La simple positive ; & divergente active : & 1 élément. \\
\hline La simple négative ; & divergente passive : & 1 élément. \\
\hline La composée négative ; & convergente inverse : & 2 éléments. \\
\hline
\end{tabular}

La première et la seule qui soit pleine liberté a été décrite. Elle développe les deux éléments attraction et raison en sens positif, ou concert de toutes deux pour la distribution et le raffinement des plaisirs. Une telle raison est convergente avec l'attraction. Il est bien peu de CIVILISÉs qui puissent exercer en ce genre, car il faut réunir santé, richesse, prudence et sûreté.On trouve quelques épicuriens qui atteignent à ce but, au moins pour quelques temps, car il n'est pas de bonheur stable en CIVILISATION, et combien petit est le nombre de ceux 
qui arrivent à l'exercice COMPOSÉ POSITIF, sort habituel du plus pauvre des hommes en HARMONIE, où il a des chances de plaisir décuples de celles de nos épicuriens ! Qu'on se rappelle la JOURNÉE HARMONIQUE décrite en $6^{\mathrm{e}}$ touche : aucun épicurien CIVILISÉ ne peut s'assurer pour un seul jour pareil sort. Un HARMONIEN en jouirait sans cesse, avec des variantes multipliées. D'ailleurs, qu'est-ce que les chances d'amusement CIVILISÉ comparées à celles de l'HARMONIE ? On a pu en juger aux chapitres qui traitent d'amour et de gastrosophie.

La liberté en $2^{\mathrm{e}}$ exercice est la SIMPLE POSITIVE, celle qui ne repose que sur l'essor de l'attraction sans concours de raison positive. Elle conduit rapidement l'homme à sa perte. Un jeune étourdi qui dissipe follement une grande fortune, jouit de ce genre de liberté qui ne lui prépare pas de bonheur pour la suite. Il est douteux qu'il aille à quelques mois sans tomber dans de fâcheuses disgrâces et même en malheur composé. C'est donc une liberté funeste que la SIMPLE POSITIVE, et avant de parler des $3^{\mathrm{e}}$ et $4^{\mathrm{e}}$, on reconnaît déjà au parallèle des $1^{\mathrm{re}}$ et $2^{\mathrm{e}}$, qu'il n'y a de bonheur pour l'homme que dans la liberté COMPOSÉE POSITIVE, qui fait converger les deux éléments, la passion du plaisir avec une raison positive, occupée à raffiner et étendre les plaisirs, mais non pas à en modérer l'usage ; auquel cas elle devient négative, et n'est plus, au lieu d'un garant de bonheur réel, qu'un garant contre l'imminence du malheur. Il faut se rappeler ici qu'en HARMONIE, on n'a pas besoin de raison modératrice, puisque la modération naît de l'état des choses, de l'affluence des plaisirs. Dès lors, la raison n'a plus qu'à exercer en composé ou en calculs de raffinements voluptueux, c'est-à-dire en positif, en service de plaisir actif.

La liberté en $3^{\mathrm{e}}$ exercice est la SIMPLE NÉGATIVE ou emploi de raison modératrice dénuée du concours de l'attraction, et n'intervenant que pour l'entraver dans des vues de prudence. Tel est le triste sort que la philosophie et la théologie veulent nous allouer en CIVILISATION. Il y a loin de là au bonheur. Savoir se résigner à souffrir, ce n'est pas jouir. Nous ne sommes point heureux quand la raison est en essor négatif, en guerre avec l'attraction ; nous ne jouissons que lorsque toutes deux s'accordent en faveur du plaisir. Ainsi, dans un grand repas, au lieu de servir les mets en confusion, nous employons la raison qui nous apprend à classer les mets en séries de quatre groupes ou quatre services consécutifs. Une telle raison est positive, eu ce qu'elle accroît la jouissance, nous assure par ce classement des mets, 
la faculté de digérer mieux et de graduer le plaisir. Mais si l'on ne sert sur la table qu'un panier de livres philosophiques et théologiques sur la tempérance, la mortification des sens et le besoin de réprimer son appétit quand on n'a pas de quoi le satisfaire, cette raison, tout eu nous persuadant par de belles phrases, ne sera qu'un plaisir négatif en conflit avec l'attraction, qui nous fait désirer un dîner quand nous nous mettons à table. Il n'est donc rien de plus opposé au bonheur que cette raison négative, à laquelle on façonne si bien les CIVILISÉs et qu'on leur donne pour libre arbitre, quoiqu'elle ne soit que la liberté de s'habituer à souffrir et guerroyer contre soi-même.

La liberté en $4^{\mathrm{e}}$ exercice est la COMPOSÉE NÉGATIVE ou ralliement des deux éléments frustrés. Le peuple CIVILISÉ ne connaît que cette quatrième liberté. Sa raison brute, mais juste, ne lui montre que malheurs dans sa misérable condition. En vain la théologie et la philosophie interviennent pour lui prouver : l'une, que ses privations sont le chemin du ciel ; l'autre, qu'elles sont le bonheur du sage. Le peuple n'entend goutte à ce grimoire de subtilités, et s'écrie partout qu'il est bien malheureux, qu'il voudrait être riche pour se livrer aux plaisirs. La philosophie lui répond que l'or et l'argent sont de vils métaux ; la théologie, qu'il n'a besoin que de la grâce et des indulgences. Il regimbe de plus belle contre ces doctes leçons, il persiste à désirer les richesses et à déplorer ses privations.

Ici les deux éléments reviennent à l'accord, mais en négatif. La raison n'est plus contraire au vœu de l'attraction comme dans la troisième liberté. L'attraction n'est plus dénuée de raison comme dans la deuxième liberté. Le peuple a vraiment la raison positive tendant à raffiner les plaisirs, car il ne désire pas la fortune pour la dissiper follement, mais pour en jouir judicieusement comme le font les gens du peuple qui s'enrichissent. On n'en voit pas un sur cent qui consume en prodigalités ce qu'il a péniblement amassé. Le peuple a donc véritablement la raison positive, ou amie des plaisirs réels sagement distribués, amie de l'attraction positive ; mais cet accord des deux éléments n’a chez lui aucun aliment : il n'opère qu'en sens négatif, puisqu'au lieu de conduire aux jouissances il aigrit le sentiment des privations.

Nous pouvons, dans les quatre exercices du libre arbitre, remarquer le contact des extrêmes; le peuple, par double engorgement de l'attraction positive et de la raison positive, se trouve de concert inten- 
tionnel avec le sybarite $\mathrm{n}^{0} 1$ qui jouit de la liberté composée positive. Cet effet s'accorde avec le principe que deux négations valent une affirmation, et que deux quantités négatives multipliées donnent le positif.

En comparant les quatre libertés, on voit qu'il n’y a de raisonnable que les deux composées : tant il est vrai que le simple n'a aucune convenance avec la nature de l'homme.

Voici matière à une belle réplique des philosophes et des théologiens, qui vont soutenir la troisième liberté, la simple négative. Puisque l'immense majorité des humains est condamnée aux privations, ne vaut-il pas mieux, diront-ils, façonner le peuple à une souffrance nécessaire, et armer la raison contre l'attraction et l'aiguillon du plaisir, que d'encourager les misérables dans leurs jérémiades et leur vaine convoitise qui est pour eux un ver rongeur? N'est-il pas plus sage de les habituer à des privations inévitables ? Non certes; le parti serait fort sage s'il n'existait pas de remède à leurs misères, point d'issue de CIVILISATION; mais il en existe douze, non compris l'ISSUE PIVOTALE ou CALCUL DE L'ATTRACTION ; et quand les deux sciences philosophie et théologie adoptent pour système de façonner le peuple au malheur, elles paralysent le génie chez la multitude et chez les savants mêmes ; elles ferment toute voie à la recherche des douze issues dont il eût été facile de découvrir quelqu'une si l'on s’en fût occupé.

Ainsi tout ce qui nous paraît sagesse relativement à la CIVILISATION devient déraison quand on spécule sur la carrière extrograde, sur les sociétés qui restaient à découvrir.

Nous pouvons, à l'aide de ces quatre modes en exercice de liberté, réduire à leur juste valeur les sornettes que nos content nos sectes savantes sur les libertés diverses, droits politiques, libre arbitre, etc. Nous allons en faire trois applications: une de la compétence des théologiens, une de celle des philosophes, une en commun aux deux classes. Ce sont de petits exemples pour exercer les élèves, comme ces analyses raisonnées qu'on donne pour tâche aux écoliers des basses classes. 


\section{COMPÉTENCE THÉOLOGIQUE}

Un homme et une jeune fille désirent se livrer au péché de fornication. Tous deux vont consulter un confesseur qui leur interdit sévèrement cette accointance, et les menace de brûler éternellement s'ils cèdent à l'attraction ou seulement au désir de commettre le péché.

D’autre part, la raison positive (raison qui coopère efficacement au plaisir) leur dit qu'en se livrant secrètement à la fornication ils ne feront de tort à personne, puisqu'aucun d'eux n'est engagé par un serment on contrat envers qui que ce soit.

Ces deux êtres jouissent-ils de la liberté et du libre arbitre ? A quel degré en sont-ils pourvus ou privés ? S'ils suivent les avis du casuiste, ils ne jouissent que du $3^{\mathrm{e}}$ degré, liberté simple négative, bornée au seul exercice de la raison ou sagesse en conflit avec l'attraction. Ils seront obligés, selon la raison, de se persuader qu'il est doux de renoncer à une fornication désirée, dont personne ne souffrirait aucun dommage. L'attraction leur dit, au contraire, qu'il serait plus doux de forniquer en secret, et que dans ce cas il y aurait exercice de liberté composée positive, ou de raison convergente avec l'attraction et intelligente à servir activement le plaisir. Jusqu'à leur décision, il y a lutte bien établie entre l'attraction et la raison ; et si l'on exige le règne de la dernière, la résistance au désir de forniquer, c'est réduire les deux individus au troisième exercice de liberté, au simple négatif, qui est un état très malheureux ainsi que je l'ai démontré.

Le libre arbitre, selon la théologie, est donc limitée à un seul des quatre exercices de la liberté, au troisième, qui est le plus fâcheux de tous. On est moins malheureux selon le quatrième, qui laisse la consolation de se plaindre et de n'admettre la raison qu'en perspective de coïncidence avec l'attraction et de service positif. Mais la théologie et la philosophie ne veulent que le troisième exercice. Elles exigent qu'on ajoute à la privation du plaisir le simulacre de la satisfaction, comme l'enfant à qui l'on donne les étrivières pour avoir mangé un morceau de sucre, et qu'on oblige ensuite à baiser le fouet respectueusement, et remercier de ce qu'on l'a fustigé pour son bien. Voilà ce qu'on appelle en CIVILISATION liberté et libre arbitre d'opter pour le bien ou le mal. Car on dira à cet enfant qu'il est libre de mal faire le 
lendemain, sauf à recevoir de nouvelles fustigations. On lui dira au besoin qu'il n'a pas le droit de manger un morceau de sucre, même en consentant aux étrivières, et pourtant cet enfant jouit, dit-on, du libre arbitre d'option pour le bien ou le mal. On avouera du moins qu'il ne jouit que de la liberté de troisième degré, simple négative.

\section{COMPÉTENCE PHILOSOPHIQUE}

Un pauvre paysan possède une somme de dix écus amassés à force d'économies et de fatigues. Un percepteur vient lui demander ces dix écus et le somme de payer avec joie, suivant la morale d'un philosophe de Paris, François de Neufchâteau, qui, dans son tableau des Devoirs du citoyen, nous dit : "Payez donc les impôts avec joie, c'est le mieux employé de l’argent que vous dépensez. »

Le paysan répond qu'il ne veut pas donner ses dix écus et qu'il ne trouve aucun plaisir à payer. Là-dessus on entremet les gendarmes et garnisaires, qui lui prouvent que le vrai bonheur est de payer les impôts. Il paie pour se débarrasser d'eux, et s'il va se plaindre de cette violence à un philosophe, celui-ci lui prouvera qu'il doit s'estimer heureux d'avoir donné toutes ses épargnes pour le bien du commerce et de la Charte, et qu'il jouit de la vraie liberté. Le paysan donne au diable ce bonheur philosophique, et soutient qu'il aurait été plus heureux et plus libre en gardant ses dix écus.

De quelle liberté a-t-il joui dans cette lutte ? C’est encore de la troisième ou conflit de la raison avec l'attraction. Il ne lui est pas permis de jouir de la quatrième, de se plaindre hautement, exprimer ce que la raison et l'attraction lui inspirent contre les sangsues publiques : on l'arrêterait comme perturbateur. Il est donc forcé de dissimuler et laisser croire qu'il a payé par raison, par amour de la morale, du commerce et de la Charte.

Dans ces deux exemples, on voit que le monde CIVILISÉ tend au $4^{\mathrm{e}}$ exercice de liberté, composée négative, à la faculté de jouir en secret ou de se plaindre hautement des entraves qu'on oppose à l'attraction. Les beaux esprits le réduisent à feindre qu'il jouit de la $3^{\mathrm{e}}$ liberté, simple négative, et qu'il trouve son bonheur à réprimer ses passions. Plai- 
sante situation pour un être qu'on dit pourvu de libre arbitre! Loin de lui laisser le droit d'opter, on ne lui laisse pas même le droit de se plaindre quand on a violenté son choix.

\section{COMPÉTENCE DES DEUX SCIENCES}

Deux conscrits sont obligés de partir, l'un en 1812, l'autre en 1818. Tous deux ont résisté jusqu'à ce que le gendarme soit venu les prendre et les conduire enchaînés. Le premier, le 1812, rencontre en chemin un philosophe qui lui dit :

« Tu es bien heureux, tu vas mourir pour la personne sacrée de Bonaparte : C’est le sort le plus beau, le plus digne d'envie ! »

À quoi le captif répond :

« J'aimerais bien mieux rester libre.

— Eh ! lui dit le philosophe, tu jouis de la vraie liberté suivant les droits de l'homme et la constitution sanctionnée par le sénat conservateur.

— Mais, si je suis libre, qu'on m'ôte ma chaîne et qu'on me laisse partir.

- Non pas, dit le philosophe, il faut, pour la liberté, que tu ailles, bon gré, mal gré, moissonner des lauriers et te faire casser les bras.

— Je ne comprends rien à cette liberté.

- Cela est bien aisé à comprendre ; il suffit de savoir que nos sensations naissent de nos perceptions par la cognition de la volition des droits de l'homme, qui font que le vrai républicain doit verser son sang pour le bien du commerce, et se doit à sa patrie.

— Eh bien! Je ne suis pas républicain, je demande qu'on me relâche.

- Comment, malheureux ! Tu ne veux pas être républicain, tu ne sens pas ta dignité d'homme libre?

— Je sens que je ne suis pas du tout libre avec mes chaînes. 
- Mais les chaînes sont douces quand on les porte pour la patrie, pour la personne sacrée de Bonaparte à qui nous devons tout notre amour. Demande plutôt au sénat conservateur.

- Bah! le sénat vit au large : c'est une troupe de sangsues qui nous grugent ; ils raisonnent à leur aise de la liberté.

- Tu parles en rebelle; si tu ne changes pas de ton, tu seras enfermé dans une forteresse et au besoin envoyé à la guillotine. Gendarmes, surveillez ce coquin-là. On te morigénera, drôle que tu es : on t’apprendra à respecter la liberté et la philosophie.

- Cela suffit, M. le philosophe, je connais maintenant votre doctrine et je crois aux perceptions de sensation et à la cognition de volition des droits de l'homme.

Le conscrit de 1818, frère du premier et conduit enchaîné par un sbire à cheval, est accosté en chemin par un théologien qui lui dit :

« Mon pauvre garçon, te voilà bien dans l'embarras, mais tu as un bon parti à prendre.

Le conscrit (à voix basse) :

«Qu'est-ce ? As-tu quelque moyen de me faire échapper au gendarme?

— Non vraiment, à Dieu ne plaise ! Je veux dire que tu peux tirer parti de la circonstance et jouir de la liberté de l'âme à défaut de celle du corps.

— Voyons si cela pourra me servir, dis-moi ton secret.

— Le voici : tu as le libre arbitre d'opter pour le bien ou pour le mal.

— Eh bien! J'opte pour m’en aller, fais-moi ôter ma chaîne.

— Pas de ça, tu opterais pour le mal en désobéissant aux lois.

— Bah ! Je veux opter pour le mal et m'en aller.

— Comment, infâme ! Tu oses préférer le mal au bien !

— Eh ! Tu me dis que je suis libre de choisir. 
— Oui, mais si tu choisis le mal, tu mérites punition.

- À quoi sert donc ta recette, s'il faut que j'opte pour rester enchaîné ; autant vaut n’avoir pas le choix.

— Tu es dans l'erreur : ce choix t'est très utile, car si tu te résignes à la volonté de Dieu et à l'obéissance aux lois, tu acquiers des mérites auprès de Dieu ; tu entres dans la voie du salut et du bonheur éternel ; tu sauves ton âme, qu'importe le corps ?

— Eh bien ! Si le corps n'est rien, mets le tien à ma place, tu auras tout le profit, une belle occasion de sauver ton âme.

— Non, j'ai d'autres fonctions à remplir, il faut que je veille au salut de mes ouailles.

— Et tu ne sais pas d'autre voie de salut pour moi ? Quelque moyen d'être libre?

— Je te mets au chemin de la vraie liberté, qui est celle de l'âme. Fais un saint usage de ton libre arbitre et résigne-toi à la volonté de Dieu.

— Chansons que tout cela! Mon frère a été tué en 1812 pour Bonaparte, et je ne suis pas tenté de faire de même.

— Ton frère était un brigand qui servait l'usurpateur, il a mérité la mort.

- Mais on l'a entraîné comme moi, enchaîné. Je serai donc un brigand selon ceux qui viendront dans 6 ans. père.

- Non, car tu vas te faire tuer pour la légitimité, pour un tendre

— Merci de sa tendresse ! J'aimerais mieux rester chez moi.

- Comment, coquin, tu n'aimes pas à te faire tuer pour l'autorité légitime ! Tu n’as point de religion ! Tu brûleras éternellement.

- Ce n'est donc pas assez que je sois torturé en ce monde, tu veux encore me brûler en l'autre. 
- Sans doute, si tu ne veux pas user de ton libre arbitre selon la volonté de Dieu et des lois. Reviens à la raison et opte pour louer Dieu du malheur par lequel il t’éprouve.

- Va-t'en jongleur, tu te moques de ma misère avec ton libre arbitre. Donne-moi plutôt un esclavage qui me fasse ôter mes chaînes. Allez, charlatans, il n'y a de libre arbitre que pour vous qui nous faites tuer en ce monde et brûler dans l'autre. »

Procédons maintenant au tarif de toutes ces libertés.

Il résulte que la première, la seule véritablement digne du nom de liberté, n'existe qu'en dose infiniment petite, que pour quelques sybarites privilégiés, à peine un homme sur mille.

Que la deuxième, qui est une liberté d'insensé et très incomplète, puisqu'elle ne se compose que d'attraction sans raison, peut s'étendre au plus à un homme sur cent.

Que la troisième, vraie servitude, lors même qu'elle paraît spontanée, vrai fardeau, puisque la plupart de ces philosophes résignés à la pauvreté accepteraient volontiers une place de 20000 francs de rente ; la troisième, dis-je, ne peut convenir qu'à des vieillards hypocrites, forcés à s’affubler de raison par la privation des moyens de jouissance.

Que la quatrième, triste consolation des malheureux CIVILISÉs, leur est encore contestée par la philosophie et la théologie, qui veulent qu'on paye avec joie, qu'on s'enrôle avec joie, qu'on renonce avec joie à tous les plaisirs, qu'on supporte avec joie toutes les vexations et qu'on feigne de jouir de la liberté simple négative, troisième, quand on n’a réellement pour lot secret que la quatrième, composée négative.

$\mathrm{Si}$, après cette analyse, quelques CIVILISÉS, autres que ceux de la classe opulente, persistent à se croire pourvus de liberté et de libre arbitre, j’y souscris, ne disputons pas des goûts. Je me borne à les renvoyer au tableau d'une journée heureuse donnée (aux Cis-Légomènes, III, 194), où l'on peut voir quel est, selon les lois de la nature, le sort d'un homme ou d'une femme réellement pourvus du libre arbitre ou raison positive convergente avec l'attraction et le plaisir. 
Qu'est-ce que les libertés d'un roi civilisé auprès de ce genre de vie assuré à perpétuité au plus pauvre des HARMONIENS ? Nos rois ne jouissent la plupart du temps que de la troisième liberté, simple négative. L'étiquette les harcèle sans cesse et les oblige à feindre du contentement quand ils sont dévorés d'ennui. Souvent leur caractère n'est point compatible avec ces servitudes, et s'ils veulent en secret se donner, comme Louis XV, quelques petits plaisirs, tels que le sérail du Parc-aux-Cerfs, ils sont traités de monstres par la philosophie et par la théologie. Les reines sont bien plus comprimées encore, et la classe des têtes couronnées n'a pas, à beaucoup près, les libertés dont jouit un riche capitaliste. Elle a d'autres avantages en essor d'ambition, mais qu'est-ce que la liberté, le libre arbitre, sinon l'essor des douze passions ? Et quelle liberté existe-t-il pour celui en qui l'étiquette ou le défaut de fortune entravent une des douze qui peut être sa dominante, celle au développement de laquelle est attaché son bonheur? 